\title{
A Framework for the Magnetic Dipole Effect on the Thixotropic Nanofluid Flow Past a Continuous Curved Stretched Surface
}

\author{
Noor Saeed Khan 1,2 ${ }^{\mathbb{D}}$, Auwalu Hamisu Usman ${ }^{3,4} \mathbb{D}$, Arif Sohail ${ }^{5}$, Abid Hussanan ${ }^{2}$, Qayyum Shah ${ }^{6}$, \\ Naeem Ullah ${ }^{7}$, Poom Kumam ${ }^{1,8, *(\mathbb{D})}$, Phatiphat Thounthong ${ }^{9}$ and Usa Wannasingha Humphries ${ }^{3, *}$
}

1 Center of Excellence in Theoretical and Computational Science (TaCS-CoE), Science Laboratory Building, Faculty of Science, King Mongkut's University of Technology Thonburi (KMUTT), 126 Pracha-Uthit Road, Bang Mod, Thrung Khru, Bangkok 10140, Thailand; noor.saeed@ue.edu.pk

2 Department of Mathematics, Division of Science and Technology, University of Education, Lahore 54000, Punjab, Pakistan; abid.hussanan@ue.edu.pk

3 Department of Mathematics, Faculty of Science, King Mongkut's University of Technology Thonburi, 126 Pracha Uthit Road, Bang Mod, Thung Khru, Bangkok 10140, Thailand; ahu3802@gmail.com

4 Department of Mathematical Sciences, Faculty of Physical Sciences, Bayero University, Kano 700241, Nigeria

5 Department of Mathematics, Khushal Khan Khattak University, Karak 27200, Khyber Pakhtunkhwa, Pakistan; arif.sohail.khattak@gmail.com

6 Department of Basic Sciences and Islamiyat, University of Engineering \& Technology, Peshawar 2500, Khyber Pakhtunkhwa, Pakistan; qayyumshah@uetpeshawar.edu.pk

7 Department of Mathematics Islamia College University, Peshawar 25000, Khyber Pakhtunkhwa, Pakistan; naeemullah1989@gmail.com

check for updates

Citation: Khan, N.S.; Usman, A.H.; Sohail, A.; Hussanan, A.; Shah, Q.; Ullah, N.; Kumam, P.; Thounthong, P.; Humphries, U.W. A Framework for the Magnetic Dipole Effect on the Thixotropic Nanofluid Flow Past a Continuous Curved Stretched Surface. Crystals 2021, 11, 645. https:// doi.org/10.3390/cryst11060645

Academic Editors: Ingo Dierking and Borislav Angelov

Received: 4 April 2021

Accepted: 24 May 2021

Published: 7 June 2021

Publisher's Note: MDPI stays neutral with regard to jurisdictional claims in published maps and institutional affiliations.

Copyright: (c) 2021 by the authors. Licensee MDPI, Basel, Switzerland. This article is an open access article distributed under the terms and conditions of the Creative Commons Attribution (CC BY) license (https:// creativecommons.org/licenses/by/ $4.0 /)$
8 Department of Medical Research, China Medical University Hospital, China Medical University, Taichung 40402, Taiwan

9 Renewable Energy Research Centre, Department of Teacher Training in Electrical Engineering, Faculty of Technical Education, King Mongkut's University of Technology North Bangkok, 1518 Pracharat 1 Road, Wongsawang, Bangsue, Bangkok 10800, Thailand; phatiphat.t@ieee.org

* Correspondences: poom.kum@kmutt.ac.th (P.K.); usa.wan@kmutt.ac.th (U.W.H.)

\begin{abstract}
The magnetic dipole effect for thixotropic nanofluid with heat and mass transfer, as well as microorganism concentration past a curved stretching surface, is discussed. The flow is in a porous medium, which describes the Darcy-Forchheimer model. Through similarity transformations, the governing equations of the problem are transformed into non-linear ordinary differential equations, which are then processed using an efficient and powerful method known as the homotopy analysis method. All the embedded parameters are considered when analyzing the problem through solution. The dipole and porosity effects reduce the velocity, while the thixotropic nanofluid parameter increases the velocity. Through the dipole and radiation effects, the temperature is enhanced. The nanoparticles concentration increases as the Biot number and curvature, solutal, chemical reaction parameters increase, while it decreases with increasing Schmidt number. The microorganism motile density decreases as the Peclet and Lewis numbers increase. Streamlines demonstrate that the trapping on the curved stretched surface is uniform.
\end{abstract}

Keywords: magnetic dipole effect; thixotropic nanofluid; curved stretching surface; analytical solution

\section{Introduction}

Non-Newtonian fluid flows have already captivated the attention of researchers. These materials are used extensively in bioengineering, geophysics, pharmaceuticals, chemical and nuclear industries, polymer solutions, cosmetics, oil storage engineering, paper manufacturing, and other fields. Clearly, no single constitutive relationship can account for all non-Newtonian materials based on behavioral shear stresses. It is distinct from Newtonian and creeping viscous fluids [1]. As a result, several non-Newtonian fluid models have been proposed [2-5]. One such model is the thixotropic fluid model. The shear thinning fluid differs from the thixotropic fluid in that the shear thinning fluid has 
less viscosity and its shear rate increases over time, whereas the viscosity of a thixotropic fluid decreases with a constant shear rate. A few studies on thixotropic and non-Newtonian fluid models can be found in the references [6-21].

The suitability of the flow of porous media for a wide range of practical industrial applications, including crude oil extraction, food storage, fossil fuels, geothermal systems, porous insulation, packaged beds, petroleum technology and waste disposal, etc., has attracted considerable interest among scientists. Various models, such as Darcy-Forchheimer and Darcy and Brinkman, have been introduced in the literature. Researchers are interested in studying porous media models because of its importance. These models may be established in the light of Darcy's law, where the pressure gradient is directly related to the average velocity of the volume. Darcy's formula may be slow and porous with no effect of inertia, porosity variable, solid boundary or thermal dispersion. To achieve the desired accurate results, the presence of non-Darcian effects is crucial for the porous media analysis as discussed by Nield and Bejan [22]. These effects are presented by Forchheimer [23], with response of the square velocity term to the Darcian velocity term. Subsequently, Morris [24] coined the term "Forchheimer", applicable to the high Reynolds number. Kishan and Maripala [25] investigated the effects of viscous dissipation and thermophoresis analysis on the mixed convection in Darcy-Forchheimer MHD fluid via porous saturated media. Rauf et al. [26] investigated the thermal radiation viscous fluid flow in Darcy-Forchheimer porous space over a curved moving surface. Jagadha [27] studied the Darcy-Forchheimer mixed convection MHD boundary layer flow with viscous dissipation in nanofluid saturated with porous media.

The ferrofluids describe a specific category of magnetizable fluids with interesting effects that have a tremendous technological impact. The ferrofluid is often a single magnetic particle domain colloidal suspension with a size of approximately $10 \mathrm{~nm}$. Aerodynamics and computer peripherals, avionics, cooling agents, crystal processing, filtration, fiber optics, loudspeakers, laser based operational devices, nuclear power plants, robotics, semiconductor processing, refrigeration, plastic drawing, etc. regularly use ferrofluids in a number of industrial applications. Like these innumerable applications, a number of studies of ferrofluids have been carried out by researchers and scientists. Andersson [28] investigated the ferrofluid with special effects of magnetic dipole. Hayat et al. [29] investigated the magnetic dipole effect on radiative ferromagnetic Williamson fluid flow. Some important studies in connection with ferrofluid are presented in the references [30-32].

For industrial, chemical applications and bio-engineering, such as drying, energy transport between desert coolers and cooling towers, food processing, production of polymers, evaporation, and metal work, the study of chemical reactions (productive/destructive) are essential for stretching surfaces. Extrusion on the stretched surface, heat transfer in the MHD stagnation point flow under the effect of chemical reactions and transpiration are analyzed by Mabood et al. [33]. Narayana and Babu [34] presented a study of MHD Jeffrey fluid flow with the chemical reaction effects over a stretching sheet numerically. Mixed peristaltic convective flow of Prandtl fluid to Hall current and chemical reaction effects is investigated by Hayat et al. [35]. Hayat et al. [36] studied the hydromagnetic flow of viscous fluid with chemical reaction and thermal radiation through a curved stretching sheet. The others relevent and stretching surfaces studies can be seen in the references [37-43].

Bioconvection is a common phenomenon that occurs in suspensions due to the upswimming of microorganisms that have a marginally higher density than water. When the upper surface of the suspensions becomes too dense due to microorganism proliferation, it becomes porous and microorganisms collapse, resulting in bioconvection. These microorganisms may exhibit gravitaxis, gyrotaxis, or oxytaxis. Supporting gyrotactic microorganisms for fluids aids in mass conversion, mixing micro-scales, and increasing fluid stability, particularly in micro-volumes. A number of researchers have investigated its various effects on fluid flow. Chamkha et al. [44] investigated the radiating effects on gyrotactic microorganisms on a vertical plate with fluid variability of temperature in natural bioconvection flow. Raju and Sandeep [45] proposed a mathematical model to 
study bioconvection through the use of non-linear chemical and thermal radiation in a rotational fluid. Hady et al. [46] studied the unsteady bioconvection thermal boundary layer flow in the presence of gyrotactic microorganisms on a stretching plate and a vertical cone in a porous medium. Recent investigations on bioconvection can be found in the references [47-56].

The current study discusses the magnetic dipole effect on thixotropic fluid with heat and mass transfer, as well as microorganism concentration passing through a curved stretching surface. The Darcy-Forchheimer model is used to describe the flow in a porous medium. Thermal radiation and viscous dissipation effects are also taken into consideration. Through appropriate similarity transformations, partial differential equations are transformed into ordinary differential equations and solved using a well-known technique, namely homotopy analysis method HAM [57-59]. Many researchers [40,47,60-63] have used HAM to solve their research problems. The results obtained are used to discuss graphically the effects of all the relevant parameters on all dimensionless profiles.

\section{Methods}

Two-dimensional hydrodynamic incompressible ferromagnetic thixotropic nanofluid past a stretched curved sheet under the influence of magnetic dipole is considered. $x$ and $y$ are used for curvilinear coordinates. The stretching surface is curled in a radius circle $R^{\prime}$. Based on the linear velocity $u=A x$ ( $A$ is constant), the sheet is stretched in the $x$-direction and $y$-direction, which is transverse to $x$-direction. The magnetic field of strength $B_{0}$ is perpendicular to the flow direction. The surface is submerged in a non-Darcy porous medium. As the Reynolds number (due to a magnet) is smaller in the present problem, the electrical and induced magnetic fields are ignored. Convective heat and mass transfer conditions are observed. In addition, a chemical reaction of the first order is also considered.

In conjunction with the above assumptions, the boundary layer of the equations involved are governed by the following terms $[7,26,27,29,30]$

$$
\begin{gathered}
\frac{\partial\left\{\left(y+R^{\prime}\right) v\right\}}{\partial y}+R^{\prime} \frac{\partial u}{\partial x}=0 \\
\frac{u^{2}}{y+R^{\prime}}=\frac{1}{\rho} \frac{\partial p}{\partial y^{\prime}} \\
\rho\left(v \frac{\partial u}{\partial y}+\frac{R^{\prime} u}{y+R^{\prime}} \frac{\partial u}{\partial x}+\frac{u v}{y+R^{\prime}}\right)=\frac{R^{\prime}}{y+R^{\prime}} \frac{\partial p}{\partial x}+\mu\left(\frac{\partial^{2} u}{\partial y^{2}}-\frac{u}{\left(y+R^{\prime}\right)^{2}}+\frac{1}{y+R^{\prime}} \frac{\partial u}{\partial y}\right) \\
-6 R_{1}\left(\frac{\partial u}{\partial y}\right)^{2}\left(\frac{\partial^{2} u}{\partial y^{2}}\right)+4 R_{2}\left[\frac{\partial u}{\partial y} \frac{\partial^{2} u}{\partial y^{2}}\left(u \frac{\partial^{2} u}{\partial x \partial y}+v \frac{\partial^{2} u}{\partial y^{2}}\right)\right. \\
\left.+\left(\frac{\partial u}{\partial y}\right)^{2}\left(u \frac{\partial^{3} u}{\partial x \partial y^{2}}+v \frac{\partial^{3} u}{\partial y^{3}}+\frac{\partial u \partial^{2} u}{\partial y \partial x \partial y}+\frac{\partial v \partial^{2} u}{\partial y \partial y^{2}}\right)\right]-\frac{\mu S_{1}}{k_{o}} u-\frac{\rho C_{b} S_{1}}{\sqrt{k_{o}}} u^{2}+\mu_{o} M \frac{\partial H}{\partial x}, \\
\left(\rho c_{p}\right)\left(\frac{R^{\prime} u}{y+R^{\prime}} \frac{\partial T}{\partial x}+v \frac{\partial T}{\partial y}\right)=\frac{k_{T}}{y+R^{\prime}}\left[\frac{\partial T}{\partial y}+\left(y+R^{\prime}\right) \frac{\partial^{2} T}{\partial y^{2}}\right]-2 \mu R_{1}\left(\frac{\partial u}{\partial y}\right)^{4}+4 \mu R_{2} u \frac{\partial^{2} u}{\partial x \partial y}\left(\frac{\partial u}{\partial y}\right)^{3} \\
+4 \mu R_{2} v \frac{\partial^{2} u}{\partial y^{2}}\left(\frac{\partial u}{\partial y}\right)^{3}+\left(u \frac{\partial H}{\partial x}+v \frac{\partial H}{\partial y}\right) \mu_{0} T \frac{\partial M}{\partial T}-\frac{k_{T}}{y+R^{\prime}}\left(\frac{\partial q_{r}}{\partial y}\left(y+R^{\prime}\right)\right), \\
\frac{R^{\prime}}{y+R^{\prime}} u \frac{\partial C}{\partial x}+v \frac{\partial C}{\partial y}=\frac{D}{y+R^{\prime}}\left(\frac{\partial C}{\partial y}+\left(y+R^{\prime}\right) \frac{\partial^{2} C}{\partial y^{2}}\right)-K_{c}\left(C-C_{\infty}\right), \\
\left(\frac{R^{\prime}}{y+R^{\prime}}\right) u \frac{\partial N}{\partial x}+v \frac{\partial N}{\partial y}+\frac{b W_{c}}{C_{w}-C_{\infty}} \frac{\partial\left(N \frac{\partial C}{\partial y}\right)}{\partial y}=D_{m} \frac{\partial^{2} N}{\partial y^{2}},
\end{gathered}
$$


with boundary conditions

$$
\begin{aligned}
& u=A x=U_{w}(x), \quad v=0,-k_{T} \frac{\partial T}{\partial y}=h_{1}\left(T_{w}-T\right),-D \frac{\partial C}{\partial y}=k_{m}\left(C_{w}-C\right), N=N_{w} \text { at } y=0, \\
& u \rightarrow 0, \quad \frac{\partial u}{\partial y} \rightarrow 0, \quad v \rightarrow 0, \quad T \rightarrow T_{\infty}, \quad C \rightarrow C_{\infty}, \quad N \rightarrow N_{\infty}, \quad \text { as } y \rightarrow \infty,
\end{aligned}
$$

where velocity components are $(u, v)$ in the radial ( $x$-direction) and transverse (y-direction), $k_{m}$ is the mass transfer coefficient, $h_{1}$ is the convective heat transfer coefficient, $R_{1}$ and $R_{2}$ are the material constants, diffusion coefficient is $D$, constant fluid density is $\rho, k_{T}$ is the thermal conductivity, $\sigma$ is the electrical conductivity, $k_{o}$ is permeability of porous medium, the effective dynamic viscosity is $\mu$, magnetic permeability is $\mu_{o}$, heat capacitance is $\left(\rho c_{p}\right)$, first order chemical reaction parameter is $K_{c}$, microorganisms diffusion is $D_{m}$, speed of gyrotactic cell is $W_{c}, b$ is the chemotaxis, $C_{b}$ is the drag coefficient, $S_{1}$ is the porosity of porous medium, $T$ is the temperature, $C$ is the concentration, $N$ is the gyrotactic microorganisms concentration, and $C_{\infty}, T_{\infty}$, and $N_{\infty}$, respectively, stand for the nanoparticles concentration, temperature, and density of microorganisms far away from the surface.

Rosseland and Ozisik approximation allows to write the radiation heat flux $q_{r}$ with $\sigma^{*}$ Stenfan-Boltzman, and $\beta_{R}$ mean absorption coefficient [64] as:

$$
q_{r}=-\frac{4 \sigma^{*}}{3 \beta_{R}} \frac{\partial T^{4}}{\partial y}=-\frac{4 T_{\infty}^{3} \sigma^{*}}{3 \beta_{R}} \frac{\partial T}{\partial y} .
$$

Magnetic Dipole

The characteristics of the magnetic field have an effect on the flow of ferrofluid due to the magnetic dipole. Magnetic dipole effects are recognized by the magnetic scalar potential $\Phi[29]$ shown in Equation (10)

$$
\Phi=\frac{\gamma}{2 \pi} \frac{x}{x^{2}+(y+c)^{2}},
$$

where $\gamma$ stands for magnetic field strength at the source, $c$ is the distance of the line currents from the leading edge. $H_{x}$ and $H_{y}$ are taken as the components of magnetic field as shown in Equations (11) and (12)

$$
\begin{aligned}
& H_{x}=-\frac{\partial \Phi}{\partial x}=\frac{\gamma}{2 \pi} \frac{x^{2}-(y+c)^{2}}{\left[x^{2}+(y+c)^{2}\right]^{2}} \\
& H_{y}=-\frac{\partial \Phi}{\partial y}=\frac{\gamma}{2 \pi} \frac{2 x(y+c)}{\left[x^{2}+(y+c)^{2}\right]^{2}} .
\end{aligned}
$$

The magnetic field $H$ is usually proportional to the components of magnetic field $H_{x}$ and $H_{y}$, gradient along $x$ and $y$ directions respectively. It is therefore defined in Equation (13) as

$$
H=\sqrt{H_{x}^{2}+H_{y}^{2}} .
$$

It is considered that the temperature-dependent variation of magnetization $M$ is linear as shown in Equation (14)

$$
M=K_{1}\left(T-T_{\infty}\right),
$$

where $K_{1}$ identifies the coefficient of the ferromagnetic. The physical schematic of the heated ferrofluid can be seen in Figure 1. 


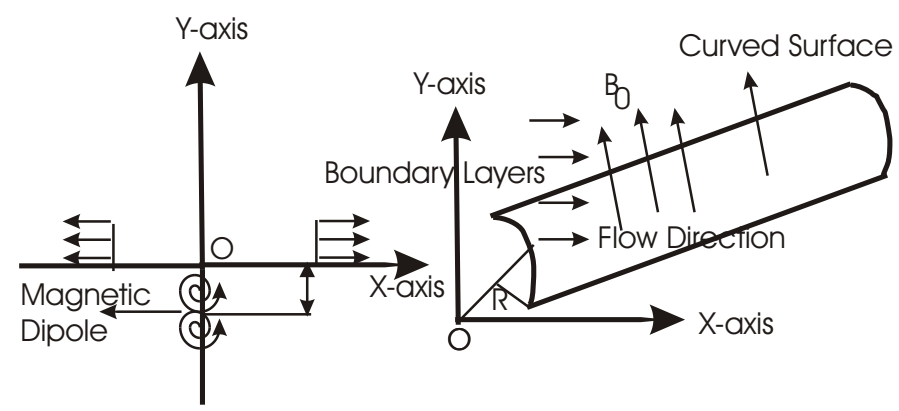

Figure 1. Schematic diagram of the problem.

Considering the following transformations [26], with $v$ as kinematic viscosity, $A$ is constant:

$$
\begin{aligned}
& u=A x f^{\prime}(\zeta), v=-\left(\frac{R^{\prime}}{y+R^{\prime}}\right) \sqrt{A v} f(\zeta), p=\rho A^{2} x^{2} p(\zeta), \quad \zeta=y \sqrt{\frac{A}{v}} \\
& \theta(\zeta)=\frac{T-T_{\infty}}{T_{w}-T_{\infty}}, \phi(\zeta)=\frac{C-C_{\infty}}{C_{w}-C_{\infty}}, \chi(\zeta)=\frac{N-N_{\infty}}{N_{w}-N_{\infty}}
\end{aligned}
$$

By the application of Equation (15), Equations (2)-(8) provide the following Equations (16), (18)-(25)

$$
\begin{gathered}
p^{\prime}=\frac{f^{\prime 2}}{\zeta+\alpha_{1}}, \\
f^{\prime \prime \prime}-\left(\frac{\alpha_{1}}{\zeta+\alpha_{1}}\right)\left[f f^{\prime \prime}-f^{\prime 2}+A_{1} f^{\prime} f\right]+N n_{1} f^{\prime \prime 2} f^{\prime \prime \prime}+N n_{2}\left[f^{\prime \prime 4}-\frac{\alpha_{1}}{\zeta+\alpha_{1}}\left(f^{\prime \prime \prime 2} f f^{\prime \prime}+f^{\prime \prime 2} f f^{\prime \prime \prime \prime}\right)\right] \\
-P_{1} f^{\prime}-L_{i} f^{\prime 2}+\frac{2 \beta}{(\zeta+d)^{4}} \theta+2\left(\frac{\alpha_{1}}{\zeta+\alpha_{1}}\right) p=0, \\
(1+R d)\left(\theta^{\prime \prime}+\frac{\theta^{\prime}}{\left(\zeta+\alpha_{1}\right)}\right)+\operatorname{Pr}\left(\frac{\alpha_{1}}{\zeta+\alpha_{1}}\right) f \theta^{\prime}+\frac{1}{3} \operatorname{Pr} N n_{1} E c f^{\prime \prime 4}+\operatorname{Pr} N n_{1} E c\left[f^{\prime} f^{\prime \prime}-f f^{\prime \prime \prime}\right] \\
+\frac{2 \beta \lambda(\theta-\epsilon) f}{(\zeta+d)^{3}}+\beta \lambda(\theta-\epsilon)\left[\frac{2 f^{\prime}}{(\zeta+d)^{4}}+\frac{4 f}{(\zeta+d)^{5}}\right]=0, \\
\phi^{\prime \prime}+\frac{\phi^{\prime}}{\left(\zeta+\alpha_{1}\right)}+\left(\frac{\alpha_{1}}{\zeta+\alpha_{1}}\right) S c f \phi^{\prime}-\delta S c \phi=0, \\
\chi^{\prime \prime}+\operatorname{Pe}\left[\phi^{\prime} \chi^{\prime}+\phi^{\prime \prime} \chi+N_{\delta} \phi^{\prime \prime}\right]+L e\left(\frac{\alpha_{1}}{\zeta+\alpha_{1}}\right) f \chi^{\prime}=0 .
\end{gathered}
$$

To eliminate the pressure term, integrating (16) to get $p$ and replacing it, then (17) becomes 


$$
\begin{aligned}
& f^{\prime \prime \prime}-\left(\frac{\alpha_{1}}{\zeta+\alpha_{1}}\right)\left[f f^{\prime \prime}-f^{\prime 2}+A_{1} f^{\prime} f\right]+N n_{1} f^{\prime \prime 2} f^{\prime \prime \prime}+N n_{2}\left[f^{\prime \prime 4}-\frac{\alpha_{1}}{\zeta+\alpha_{1}}\left(f^{\prime \prime \prime 2} f f^{\prime \prime}+f^{\prime \prime 2} f f^{\prime \prime \prime \prime}\right)\right] \\
& -P_{1} f^{\prime}-L_{i} f^{\prime 2}+\frac{2 \beta}{(\zeta+d)^{4}} \theta+\left(\frac{\alpha_{1}}{\left(\zeta+\alpha_{1}\right)^{2}}\right)\left(2 f f^{\prime \prime}-f^{\prime 2}\right)=0
\end{aligned}
$$

and the boundary conditions become

$$
\begin{aligned}
& f^{\prime}(0)=1, f(0)=0, f(\infty)=0, f^{\prime \prime}(\infty)=0, \\
& \theta^{\prime}(0)=-B i_{1}[1-\theta(0)], \theta(\infty)=0, \\
& \phi^{\prime}(0)=-B i_{2}[1-\phi(0)], \phi(\infty)=0, \\
& \chi^{\prime}(0)=1, \chi(\infty)=0,
\end{aligned}
$$

where $A_{1}$ is the ratio of rate constants, $\alpha_{1}$ is the curvature parameter, $d$ is the dimensionless distance, $N n_{1}$ and $N n_{2}$ are the non-Newtonian parameters, $\beta$ is the ferrohydrodynamic interaction parameter, heat dissipation parameter is $\lambda, \varepsilon$ is the curie temperature, Prandtl number is $P r$, radiation parameter is $R d$, Eckert number is $E c$, chemical reaction parameter is $\delta$, the Schmidt number is $S c$, local inertia parameter is $L i$, porosity parameter is $P_{1}$, Lewis number is $P e$, Lewis number is $L e$, thermal Biot number is $B i_{1}$ and concentration Biot number is $B i_{2}$, which are defined by

$$
\begin{aligned}
& A_{1}=\sqrt{\frac{\mu}{\rho A}}, \alpha_{1}=R^{\prime} \sqrt{\frac{A}{v}}, N n_{1}=\frac{4 R_{2} A^{4} x^{2}}{\rho v^{2}}, N n_{2}=\frac{-6 R_{1} A^{4} x^{2}}{\rho v}, P_{1}=\frac{\mu S_{1}}{\rho A k_{o}^{*}}, L_{i}=\frac{C_{b} S_{1}}{\sqrt{k_{o}^{*}}} \\
& \beta=\frac{\gamma \mu_{0} K_{1} \rho\left(T_{w}-T_{\infty}\right)}{2 \pi \mu^{2}}, P r=\frac{\mu C_{P}}{k_{T}}, E c=\frac{(A x)^{2}}{C_{P}\left(T_{w}-T_{\infty}\right)}, \lambda=\frac{A \mu^{2}}{\rho\left(T_{w}-T_{\infty}\right) k_{T}}, d=\sqrt{\frac{A c^{2}}{v}}, \\
& \delta=\frac{A K_{c}}{v}, R d=\frac{16 \sigma^{*} T_{\infty}^{3}}{3 k^{*}}, S c=\frac{v}{D}, P e=\frac{b W_{c}}{D_{m}}, L e=\frac{v}{D_{n}}, B i_{1}=\frac{h_{1}}{k_{T}} \sqrt{\frac{\nu}{A}}, B i_{2}=\frac{k_{m}}{k_{T}} \sqrt{\frac{\nu}{A}} \\
& \epsilon=\frac{T_{\infty}}{T_{\infty}-T_{w}}, N_{\delta}=\frac{N_{\infty}}{N_{w}-N_{\infty}} .
\end{aligned}
$$

The quantities of interest, such as coefficient of skin friction, local Nusselt, Sherwood and local density numbers, are determined by

$$
C_{f}=\frac{\tau_{y x}}{\rho(A x)^{2}}, N u_{x}=\frac{-x q_{w}}{k_{T}\left(T_{w}-T_{\infty}\right)}, S h_{x}=\frac{-x q_{m}}{D\left(C_{w}-C_{\infty}\right)}, S n_{x}=\frac{-x q_{n}}{D\left(N_{w}-N_{\infty}\right)},
$$

where

$$
\tau_{y x}=\left.\mu u_{y}\right|_{y=0}, q_{w}=-\left.k_{T} T_{y}\right|_{y=0}-\left.\frac{4 T_{\infty}^{3} \sigma^{*}}{3 \beta_{R}} \frac{\partial T}{\partial y}\right|_{y=0}, q_{m}=-\left.D C_{y}\right|_{y=0}, q_{n}=\left.D_{m} N_{y}\right|_{y=0} .
$$

By putting values from Equation (28) in Equation (27), it is obtained that

$$
\begin{aligned}
C_{f} & =\frac{1}{R e_{x}}\left(f^{\prime \prime}(0)-\frac{f^{\prime}(0)}{\alpha_{1}}\right), N u=-R e_{x}^{0.5}(1+R d) \theta^{\prime}(0), \\
S h & =-R e_{x}^{0.5} \phi^{\prime}(0), S n=-R e_{x}^{0.5} \chi^{\prime}(0) .
\end{aligned}
$$

\section{HAM Solution}

The initial guesses and the linear operators are taken as

$$
f_{0}(\zeta)=A \zeta+(1-A)\left(1-e^{-\zeta}\right), \theta_{0}(\zeta)=\frac{B i_{1}}{1+B i_{1}} e^{-\zeta}, \phi_{0}(\zeta)=\frac{B i_{2}}{1+B i_{2}} e^{-\zeta}, \chi_{0}=e^{-\zeta}
$$


Equation (30) satisfies the properties as given below

$$
\begin{aligned}
& L_{f}\left(E_{1}+E_{2} e^{\zeta}+E_{3} e^{-\zeta}\right)=0, \quad L_{\theta}\left(E_{4} e^{\zeta}+E_{5} e^{-\zeta}\right)=0, \\
& L_{\phi}\left(E_{6} e^{\zeta}+E_{7} e^{-\zeta}\right)=0, \quad L_{\chi}\left(E_{8} e^{\zeta}+E_{9} e^{-\zeta}\right)=0,
\end{aligned}
$$

where $E_{i}(i=1, \ldots, 9)$ indicates the arbitrary constants.

The corresponding zeroth order form of the problems are

$$
\begin{aligned}
& (1-q) L_{f}\left[f(\zeta, q)-f_{0}(\zeta)\right]=q h_{f} N_{f}[f(\zeta, q), \theta(\zeta, q)], \\
& (1-q) L_{\theta}\left[\theta(\zeta, q)-\theta_{0}(\zeta)\right]=q h_{\theta} N_{\theta}[\theta(\zeta, q), f(\zeta, q)], \\
& (1-q) L_{\phi}\left[\phi(\zeta, q)-\phi_{0}(\zeta)\right]=q h_{\phi} N_{\phi}[\phi(\zeta, q), f(\zeta, q)], \\
& (1-q) L_{\chi}\left[\chi(\zeta, q)-\chi_{0}(\zeta)\right]=q h_{\chi} N_{\chi}[\chi(\zeta, q), \phi(\zeta, q), f(\zeta, q)],
\end{aligned}
$$

$$
\begin{aligned}
& f(0, q)=0, f^{\prime}(0, q)=1, f^{\prime}(\infty, q)=A, \theta^{\prime}(0, q)=-B i_{1}(1-\theta(0, q)), \theta(\infty, q)=0, \\
& \phi^{\prime}(0, q)=-B i_{2}(1-\phi(0, q)), \phi(\infty, q)=0 \chi^{\prime}(0, q)=1, \chi(\infty, q)=0,
\end{aligned}
$$

$$
\begin{aligned}
& \mathbf{N}_{f}[f(\zeta, q)]=\frac{\partial^{3} f(\zeta, q)}{\partial \zeta^{3}}-\left(\frac{\alpha_{1}}{\zeta+\alpha_{1}}\right)\left[f(\zeta, q) \frac{\partial^{2} f(\zeta, q)}{\partial \zeta^{2}}-\left(\frac{\partial f(\zeta, q)}{\partial \zeta}\right)^{2}+A_{1} \frac{\partial f(\zeta, q)}{\partial \zeta} f(\zeta, q)\right] \\
& +N n_{1}\left(\frac{\partial^{2} f(\zeta, q)}{\partial \zeta^{2}}\right)^{2} \frac{\partial^{3} f(\zeta, q)}{\partial \zeta^{3}}+N n_{2}\left[\left(\frac{\partial^{2} f(\zeta, q)}{\partial \zeta^{2}}\right)^{4}-\frac{\alpha_{1}}{\zeta+\alpha_{1}}\left(\left(\frac{\partial^{3} f(\zeta, q)}{\partial \zeta^{3}}\right)^{2} f(\zeta, q) \frac{\partial^{2} f(\zeta, q)}{\partial \zeta^{2}}\right.\right. \\
& \left.\left.+\left(\frac{\partial^{2} f(\zeta, q)}{\partial \zeta^{2}}\right)^{2} f(\zeta, q) \frac{\partial^{4} f(\zeta, q)}{\partial \zeta^{4}}\right)\right]-P 1 \frac{\partial f(\zeta, q)}{\partial \zeta}-L_{i}\left(\frac{\partial f(\zeta, q)}{\partial \zeta}\right)^{2} \\
& +\frac{2 \beta}{(\zeta+d)^{4}} \theta(\zeta, q)+\left(\frac{\alpha_{1}}{\left(\zeta+\alpha_{1}\right)^{2}}\right)\left(2 f(\zeta, q) \frac{\partial^{2} f(\zeta, q)}{\partial \zeta^{2}}-\left(\frac{\partial f(\zeta, q)}{\partial \zeta}\right)^{2}\right), \\
& \quad \mathbf{N}_{\theta}[\theta(\zeta, q)]=(1+R d)\left(\frac{\partial^{2} \theta(\zeta, q)}{\partial \zeta^{2}}+\frac{1}{\left(\zeta+\alpha_{1}\right)} \frac{\partial \theta(\zeta, q)}{\partial \zeta}\right)+\operatorname{Pr}\left(\frac{\alpha_{1}}{\zeta+\alpha_{1}}\right) f(\zeta, q) \frac{\partial \theta(\zeta, q)}{\partial \zeta} \\
& +\frac{1}{3} \operatorname{PrNn} n_{1} E c\left(\frac{\partial^{2} f(\zeta, q)}{\partial \zeta^{2}}\right)^{4}+\operatorname{PrNn} n_{2} E c\left[\frac{\partial f(\zeta, q)}{\partial \zeta} \frac{\partial^{2} f(\zeta, q)}{\partial \zeta^{2}}-f(\zeta, q) \frac{\partial^{3} f(\zeta, q)}{\partial \zeta^{3}}\right] \\
& +\frac{2 \beta \lambda(\theta(\zeta, q)-\epsilon) f(\zeta, q)}{(\zeta+d)^{3}}+\beta \lambda(\theta-\epsilon)\left[\frac{2}{(\zeta+d)^{4}} \frac{\partial f(\zeta, q)}{\partial \zeta}+\frac{4 f(\zeta, q)}{(\zeta+d)^{5}}\right],
\end{aligned}
$$

$$
\begin{aligned}
& \mathbf{N}_{\phi}[\phi(\zeta, q)]=\frac{\partial^{2} \phi(\zeta, q)}{\partial \zeta^{2}}+\frac{1}{\left(\zeta+\alpha_{1}\right)} \frac{\partial \phi(\zeta, q)}{\partial \zeta}+\left(\frac{\alpha_{1}}{\zeta+\alpha_{1}}\right) \operatorname{Sc} f(\zeta, q) \frac{\partial \phi(\zeta, q)}{\partial \zeta}-\delta \operatorname{Sc}(\zeta, q), \\
& \mathbf{N}_{\chi}[\chi(\zeta, q)]=\frac{\partial^{2} \chi(\zeta, q)}{\partial \zeta^{2}}+\operatorname{Pe}\left[\frac{\partial \phi(\zeta, q)}{\partial \zeta} \frac{\partial \chi(\zeta, q)}{\partial \zeta}+\frac{\partial^{2} \phi(\zeta, q)}{\partial \zeta^{2}} \chi(\zeta, q)+N_{\delta} \frac{\partial^{2} \phi(\zeta, q)}{\partial \zeta^{2}}\right] \\
& \quad+L e\left(\frac{\alpha_{1}}{\zeta+\alpha_{1}}\right) f(\zeta, q) \frac{\partial \phi(\zeta, q)}{\partial \zeta},
\end{aligned}
$$

where $q \in[0,1]$ is the embedding parameter while $\mathbf{N}_{f}, \mathbf{N}_{\theta}, \mathbf{N}_{\phi}$, and $\mathbf{N}_{\chi}$ are the nonlinear operators.

The $m$-th order deformation problems are as follows 


$$
\begin{aligned}
& L_{f}\left[f_{m}(\zeta, q)-\eta_{m} f_{m-1}(\zeta)\right]=h_{f} \mathcal{R}_{f, m}(\zeta), \\
& L_{\theta}\left[\theta_{m}(\zeta, q)-\eta_{m} \theta_{m-1}(\zeta)\right]=h_{\theta} \mathcal{R}_{\theta, m}(\zeta), \\
& \mathcal{L}_{\phi}\left[\phi_{m}(\zeta, q)-\eta_{m} \phi_{m-1}(\zeta)\right]=h_{\phi} \mathcal{R}_{\phi, m}(\zeta), \\
& L_{\chi}\left[\chi_{m}(\zeta, q)-\eta_{m} \chi_{m-1}(\zeta)\right]=h_{\chi} \mathcal{R}_{\chi, m}(\zeta) \text {, } \\
& f_{m}(0)=f_{m}^{\prime}(0)=f_{m}^{\prime}(\infty)=0, \\
& \theta_{m}^{\prime}(0)-B i_{1} \theta_{m}(0)=\theta_{m}(\infty)=0, \\
& \phi_{m}^{\prime}(0)-B i_{2} \phi_{m}(0)=\phi_{m}(\infty)=0, \\
& \chi_{m}^{\prime}(0)=\chi_{m}(0)=\chi_{m}(\infty)=0 \text {, } \\
& \mathcal{R}_{f}^{m}(\zeta)=f_{m-1}^{\prime \prime \prime}-\left(\frac{\alpha_{1}}{\zeta+\alpha_{1}}\right)\left[\sum_{r=0}^{m-1} f_{m-1-r} f_{r}^{\prime \prime}-\sum_{r=0}^{m-1} f_{m-1-r}^{\prime} f_{r}^{\prime}+A_{1} \sum_{r=0}^{m-1} f_{m-1-r} f_{r}^{\prime}\right] \\
& +N n_{1} \sum_{r=0}^{m-1}\left(\sum_{k=0}^{r} f_{m-1-r}^{\prime \prime} f_{r-k}^{\prime \prime}\right) f_{k}^{\prime \prime \prime}+N n_{2}\left[\sum_{r=0}^{m-1}\left(\sum_{k=0}^{r}\left(\sum_{p=0}^{k} f_{m-1-r}^{\prime \prime} f_{r-k}^{\prime \prime}\right) f_{k-p}^{\prime \prime}\right) f_{p}^{\prime \prime}\right. \\
& -\frac{\alpha_{1}}{\zeta+\alpha_{1}}\left(\sum_{r=0}^{m-1}\left(\sum_{k=0}^{r}\left(\sum_{p=0}^{k} f_{m-1-r} f_{r-k}^{\prime \prime \prime}\right) f_{k-p}^{\prime \prime \prime}\right) f_{p}^{\prime \prime \prime \prime}\right. \\
& \left.\left.+\sum_{r=0}^{m-1}\left(\sum_{k=0}^{r}\left(\sum_{p=0}^{k} f_{m-1-r} f_{r-k}^{\prime \prime}\right) f_{k-p}^{\prime \prime}\right) f_{p}^{\prime \prime \prime \prime}\right)\right]-P_{1} f_{m-1}^{\prime}-L_{i} \sum_{r=0}^{m-1} f_{m-1-r}^{\prime} f_{r}^{\prime} \\
& +\frac{2 \beta}{(\zeta+d)^{4}} \theta_{m-1}+\frac{\alpha_{1}}{\left(\zeta+\alpha_{1}\right)^{2}}\left(2 \sum_{r=0}^{m-1} f_{m-1-r} f_{r}^{\prime \prime}-\sum_{r=0}^{m-1} f_{m-1-r}^{\prime} f_{r}^{\prime}\right), \\
& \mathcal{R}_{\theta}^{m}(\zeta)=(1+R d)\left(\theta_{m-1}^{\prime \prime}+\frac{\theta_{m-1}^{\prime}}{\left(\zeta+\alpha_{1}\right)}\right)+\operatorname{Pr}\left(\frac{\alpha_{1}}{\zeta+\alpha_{1}}\right) \sum_{r=0}^{m-1} f_{m-1-r} \theta_{r}^{\prime} \\
& +\frac{1}{3} \operatorname{PrN} n_{1} E c \sum_{r=0}^{m-1}\left(\sum_{k=0}^{r}\left(\sum_{p=0}^{k} f_{m-1-r}^{\prime \prime} f_{r-k}^{\prime \prime}\right) f_{k-p}^{\prime \prime}\right) f_{p}^{\prime \prime}
\end{aligned}
$$

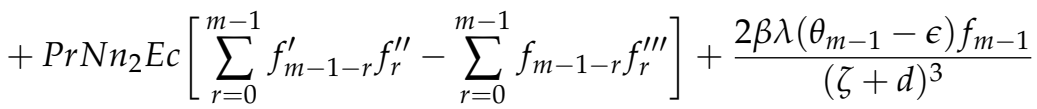

$$
\begin{aligned}
& +\beta \lambda\left(\theta_{m-1}-\epsilon\right)\left[\frac{2 f_{m-1}^{\prime}}{(\zeta+d)^{4}}+\frac{4 f_{m-1}}{(\zeta+d)^{5}}\right], \\
& \mathcal{R}_{\phi}^{m}(\zeta)=\phi_{m-1}^{\prime \prime}+\frac{\phi_{m-1}^{\prime}}{\left(\zeta+\alpha_{1}\right)}+\left(\frac{\alpha_{1}}{\zeta+\alpha_{1}}\right) S c \sum_{r=0}^{m-1} f_{m-1-r} \phi_{r}^{\prime}-\delta S c \phi_{m-1} \\
& \mathcal{R}_{\chi}^{m}(\zeta)=\chi_{m-1}^{\prime \prime}+\operatorname{Pe}\left[\sum_{r=0}^{m-1} \phi_{m-1-r}^{\prime} \chi_{r}^{\prime}+\sum_{r=0}^{m-1} \phi_{m-1-r}^{\prime \prime} \chi_{r}+N_{\delta} \phi_{m-1}^{\prime \prime}\right] \\
& +L e\left(\frac{\alpha_{1}}{\zeta+\alpha_{1}}\right) \sum_{r=0}^{m-1} f_{m-1-r} \chi_{r}^{\prime}
\end{aligned}
$$

where

$$
\eta_{m}=\left\{\begin{array}{l}
0, \text { if } m \leq 1 \\
1, \text { if } m>1
\end{array}\right.
$$


The general solutions are given by

$$
\begin{aligned}
& f_{m}(\zeta)=f_{m}^{*}(\zeta)+E_{1}+E_{2} e^{\zeta}+E_{3} e^{-\zeta}, \\
& \theta_{m}(\zeta)=\theta_{m}^{*}(\zeta)+E_{4} e^{\zeta}+E_{5} e^{-\zeta}, \\
& \phi_{m}(\zeta)=\phi_{m}^{*}(\zeta)+E_{6} e^{\zeta}+E_{7} e^{-\zeta}, \\
& \chi_{m}(\zeta)=\chi_{m}^{*}(\zeta)+E_{8} e^{\zeta}+E_{9} e^{-\zeta},
\end{aligned}
$$

where $\left(f_{m}^{*}(\zeta), \theta_{m}^{*}(\zeta), \phi_{m}^{*}(\zeta), \chi_{m}^{*}(\zeta)\right)$ are special solutions.

\section{Convergence Analysis of the Homotopy Solution}

The nonzero auxiliary parameters are involved in the homotopy solution. These parameters are extremely important in controlling and adjusting the convergence acquired by the homotopic series solutions. The $h$-curves at the 15 th order of approximations are sketched to show the acceptable approximate region of convergence. Figure 2 depicts the region as falling within the ranges $-1.8 \leq h_{f} \leq 0.2,-2.5 \leq h_{\theta} \leq 0.8,-4.2 \leq h_{\phi} \leq$ $1.1,-0.4 \leq h_{\chi} \leq 0.4$. The values of parameters used are $A_{1}=d=1, \lambda=\beta=P_{1}=$ $L i=S c=0.3, \varepsilon=\delta=E c=0.1, P e=L e=N_{\delta}=0.2, N n_{1}=N n_{2}=0.5, \operatorname{Pr}=6.8, \operatorname{Re}=$ $0.7, \alpha_{1}=R d=B i_{1}=B i_{2}=0.4$.

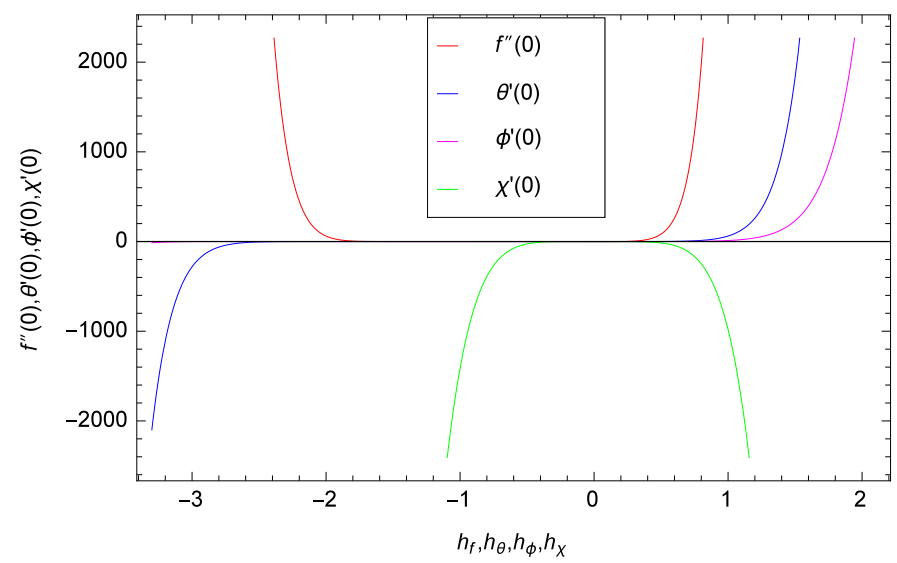

Figure 2. $h$-curves for $f(\zeta), \theta(\zeta), \phi(\zeta), \chi(\zeta)$.

\section{Discussion}

The velocity behavior with the ferromagnetic hydrodynamic interaction parameter $\beta$ can be seen in Figure 3. It demonstrates that the velocity decreases as $\beta$ increases. Ideally, the resistance force known as Lorentz force [65] increases with the $\beta$ increase, and the velocity field decreases. Figure 4 is used to investigate the effect of curvature parameter $\alpha_{1}$ on the velocity profile. It is clearly shown in the figure that the velocity component decreases for larger $\alpha_{1}$. Figures 5 and 6 describe the effects of the thixotropic parameters $N n_{1}$ and $N n_{2}$ on the velocity profile. From these figures, it is observed that $N n_{1}$ and $\mathrm{Nn}_{2}$ result in an increase in fluid velocity. Ideally, $\mathrm{Nn} n_{1}$ and $\mathrm{Nn} n_{2}$ are associated with the properties of shear thinning, which show a time-dependent changes in viscosity. The higher the fluid under shear stress, the lower the viscosity of nanofluid, which will ultimately lead to an increase in fluid velocity. Figure 7 is used to present the velocity behavior with the porosity parameter $P_{1}$. The presence of porous medium slows down the field of the flow, resulting in an increase in shear stress on the curved surface, and therefore the velocity profile shows a declining trend by increasing the values of $P_{1}$. In contrast to the effect seen with $P_{1}$, change in local inertia parameter $L i$ results in an increase in velocity as shown in Figure 8.

Figure 9 is used used to examine the effect of $\beta$ on temperature. Here, temperature increases with higher values of $\beta$. The temperature profile behavior relating to the higher values of thermal Biot number $B i_{1}$ is shown in Figure 10. The parameter $B i_{1}$ significantly 
promotes the temperature field in a positive manner attributable to the effective convective heat effects. It is also observed that there is no heat transfer at $B i_{1}=0$. The effect of the heat dissipation parameter $\lambda$ on temperature is shown in Figure 11. The temperature is a decreasing function of $\lambda$. Physically thermal conductivity of liquid decreases with larger $\lambda$, and therefore the temperature decreases. The Eckert number $E c$ attributes to the temperature profile is shown in Figure 12. For larger Ec, temperature and thermal boundary layer thickness were observed to be effected with the increase in Ec. In this phenomenon, the heat energy stored in the fluid is caused by friction forces that increase the temperature. The Curie temperature parameter $\varepsilon$ effect on temperatureprofile is shown in Figure 13. The temperature decreases through larger values of $\varepsilon$. Thermal conductivity of the liquid increases with the larger $\varepsilon$. The effect of Prandtl number $\operatorname{Pr}$ on temperature profile is shown in Figure 14. The temperature distribution and thermal boundary layer are reduced by higher values of $\mathrm{Pr}$, due to which thermal diffusion is reduced. In addition, fluids with a smaller values of $P r$ slowly decay compared to liquids with larger values of $P r$. The effect of radiation parameter $R d$ on temperature profile is discussed in Figure 15. The increase in temperature curves with a larger boundary layer thickness is determined by an increase in $R d$. Usually, mean absorption coefficient decays for higher estimation of $R d$ and diffusion flux occurs as a consequence of the temperature gradient, which therefore increases the temperature.

The effect of the concentration Biot number $\mathrm{Bi}_{2}$ on the nanoparticles concentration profile is shown in Figure 16. In this case, the concentration is increased in response to increase in the $B i_{2}$ values. Figure 17 shows the effect of the $S c$ on concentration profile. Since $S c$ is the ratio of momentum to mass diffusivity, the increase in $S c$ causes a decay in mass diffusivity, thus leading to a decrease in nanoparticles concentration. Figure 18 shows the effect of the curvature parameter $\alpha_{1}$ on the nanoparticles concentration profile. The increase in the curvature parameter results in an increase in the concentration. Figure 19 shows the effect of the chemical reaction parameter $\delta$ on the concentration profile. The nanoparticles concentration is observed to increase for the higher estimates of $\delta$. In fact, the consumption of reactive species rapidly declines as $\delta$ becomes larger.

Figure 20 shows the effect of Peclet number $P e$ on the microorganisms profile. There is a clear relationship between the reduced density of the microorganisms and the increase in $P e$. The higher values of $P e$ indicate the minimum motile diffusivity. Figure 21 shows the impact of Lewis number Le on microorganisms concentration profile. The decrease in the concentration distribution is shown as the Lewis number increases, since it is inversely proportional to the mass diffusion.

The effect of the dimensionless variable $\zeta$ on the streamlines is shown in Figures 22 and 23. It is shown that the number of the trapped boluses increases as the values of $\zeta$ increase, and the streamlines have also been identified to be perpendicular to the surface. The increase in the $\zeta$ increases the shearing motion, which, in fact, results in a higher precession of the flow to the stretching surface.

Table 1 shows a numerical analysis of the skin friction coefficient for $\beta, \alpha_{1}, P_{1}, L i, N n_{1}, N n_{2}$. It is discovered that the skin friction coefficient increases with the increasing values of $\beta, P_{1}, L i, N n_{2}$, while a reverse trend is observed for $\alpha_{1}$ and $N n_{1}$. Table 2 cross-checks the accurateness of the homotopic solution used in the present investigation. A comparison of skin friction coefficient for the different values of $\alpha_{1}$ with the study [66] is shown for $P_{1}=L i=\beta=A_{1}=N n_{1}=N n_{2}=0$ at $\zeta=0$. Table 3 shows the numerical assessment of the local Nusselt number for various values of $\beta, \alpha_{1}, \lambda, \operatorname{Pr}, R d, \varepsilon, E c, N n_{1}, N n_{2}$. It is observed that the local Nusselt number decreases with increasing values of $\beta, \alpha_{1}, \lambda, N n_{1}$. Table 4 shows the numerical values of the local Sherwood number for various values of $\alpha_{1}, S_{c}, \delta$. It is observed that the local Sherwood number decreases with the increasing values of parameters. The tables clearly show that the current findings are completely consistent. 


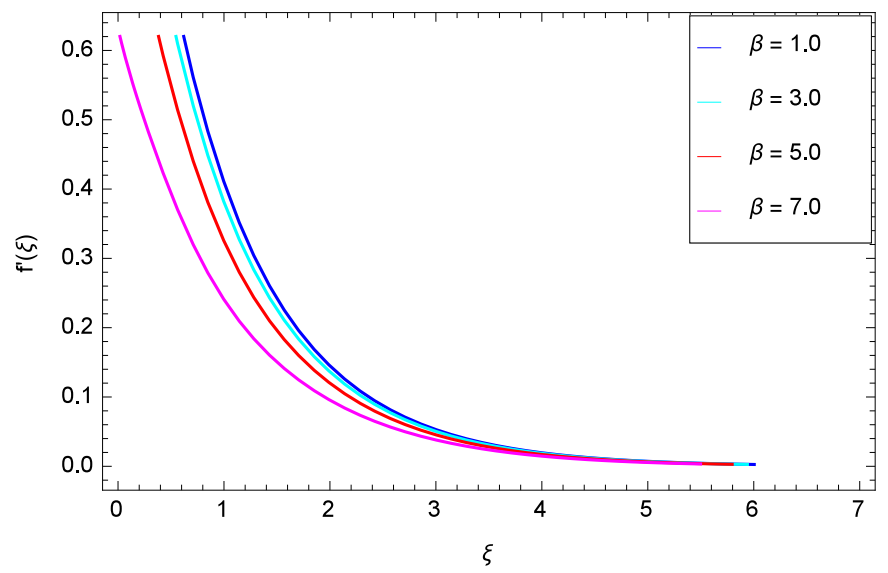

Figure 3. Impact of the ferrohydrodynamic interaction parameter $\beta$ on velocity profile $f^{\prime}(\zeta)$.

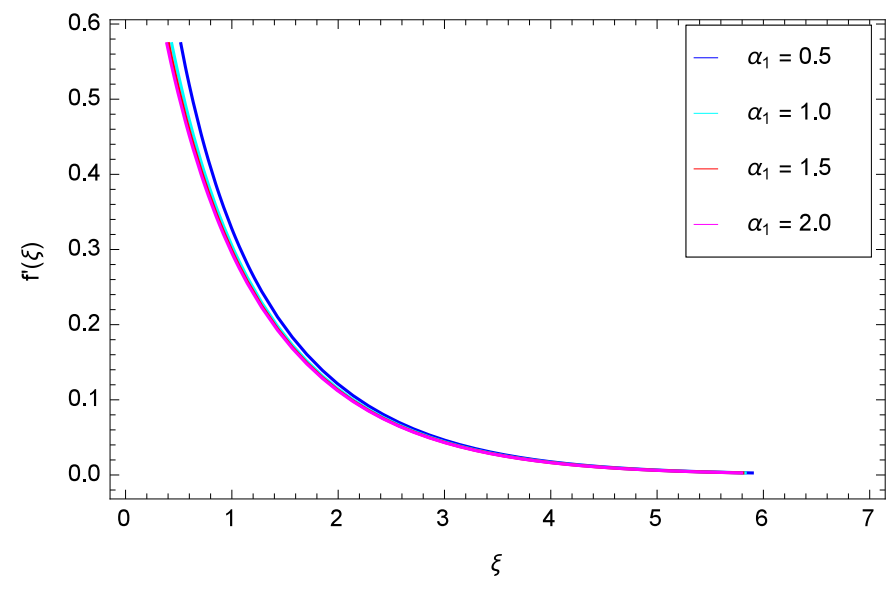

Figure 4. Impact of the curvature paraeter $\alpha_{1}$ on velocity $f^{\prime}(\zeta)$.

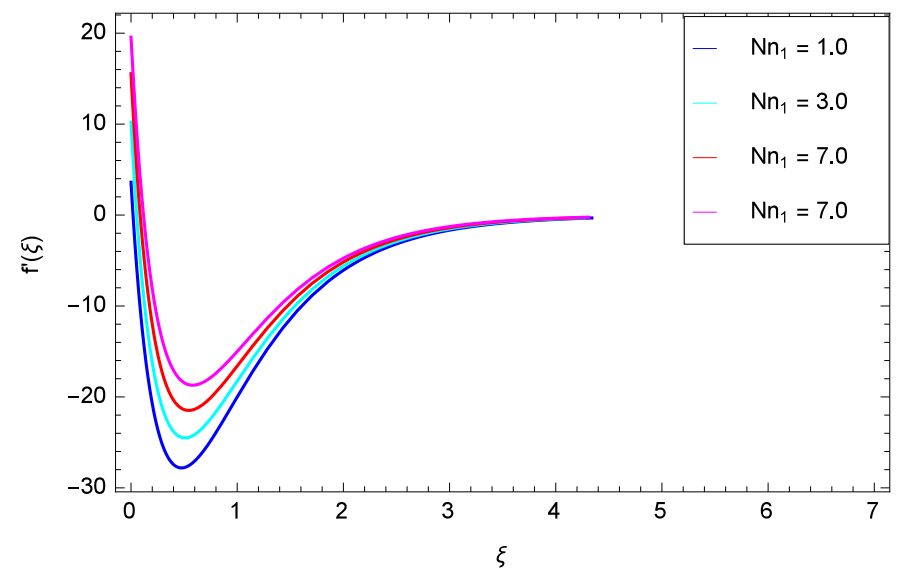

Figure 5. Impact of the non-Newtonian parameter $N n_{1}$ on velocity $f^{\prime}(\zeta)$. 


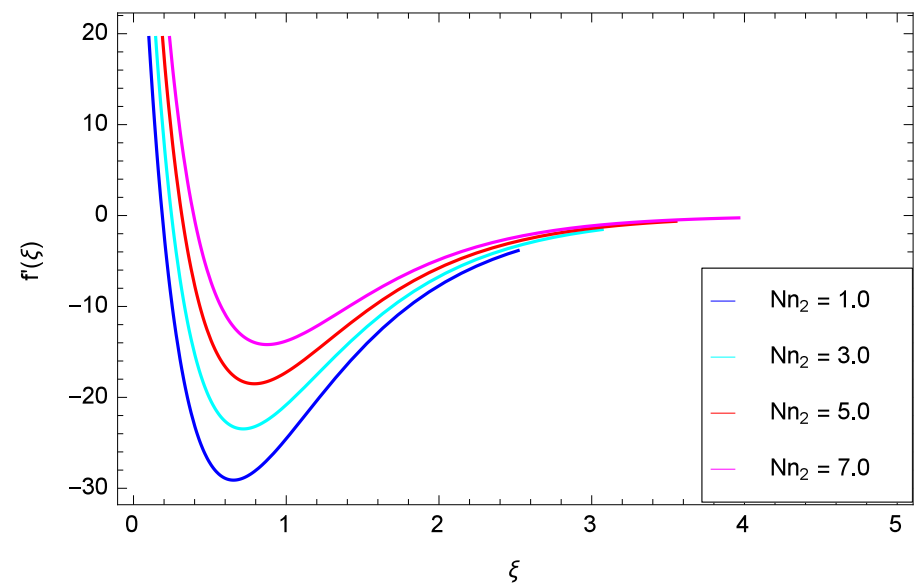

Figure 6. Impact of the non-Newtonian parameter $N n_{2}$ on velocity $f^{\prime}(\zeta)$.

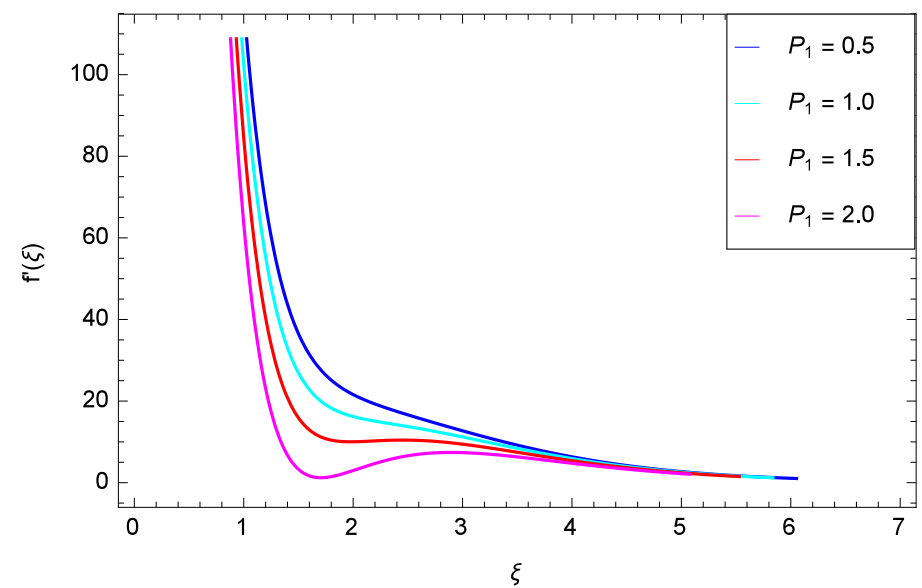

Figure 7. Impact of the porosity parameter $P_{1}$ on velocity $f^{\prime}(\zeta)$.

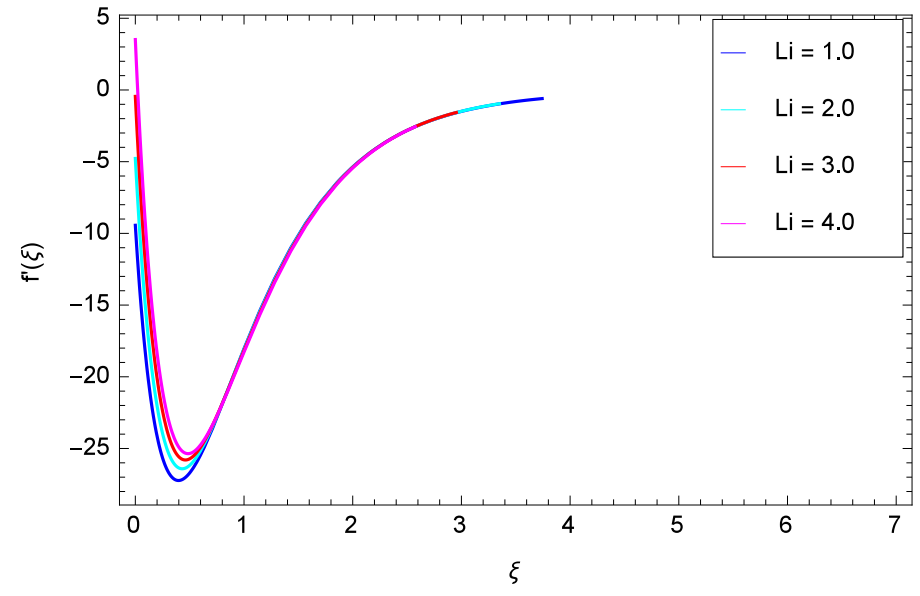

Figure 8. Impact of the local inertia parameter $L i$ on velocity $f^{\prime}(\zeta)$. 


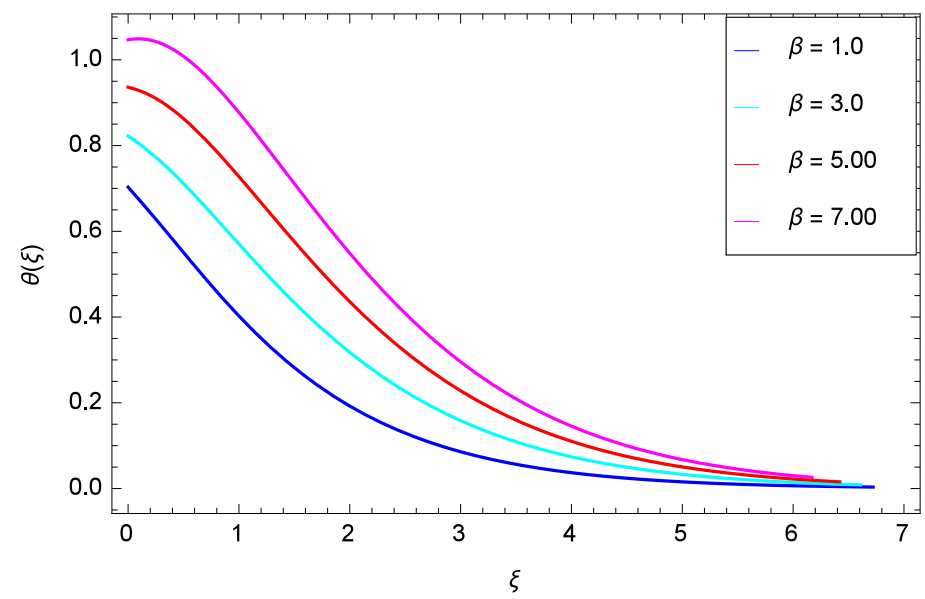

Figure 9. Impact of the ferrohydrodynamic interaction parameter $\beta$ on temperature $\theta(\zeta)$.

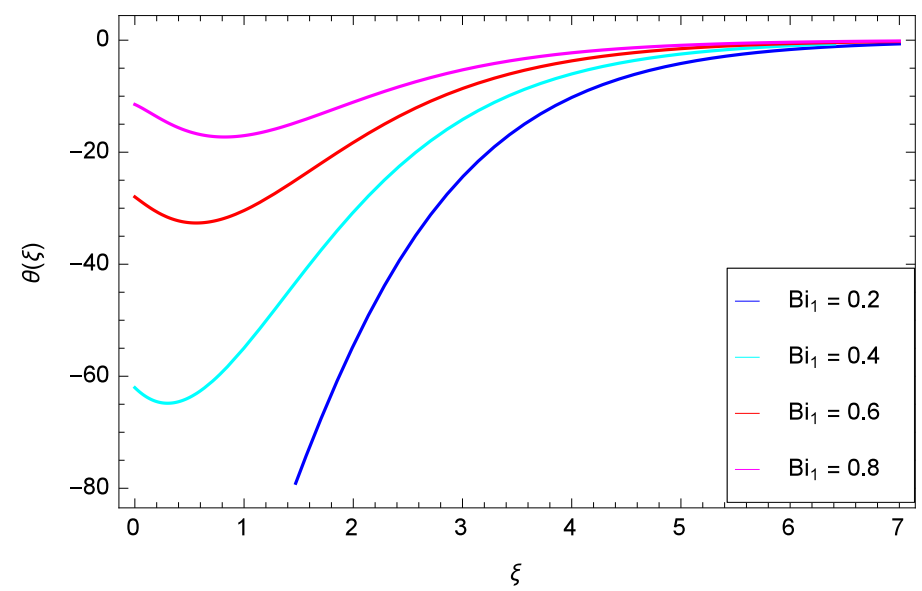

Figure 10. Impact of the thermal Biot number $B i_{1}$ on temperature $\theta(\zeta)$.

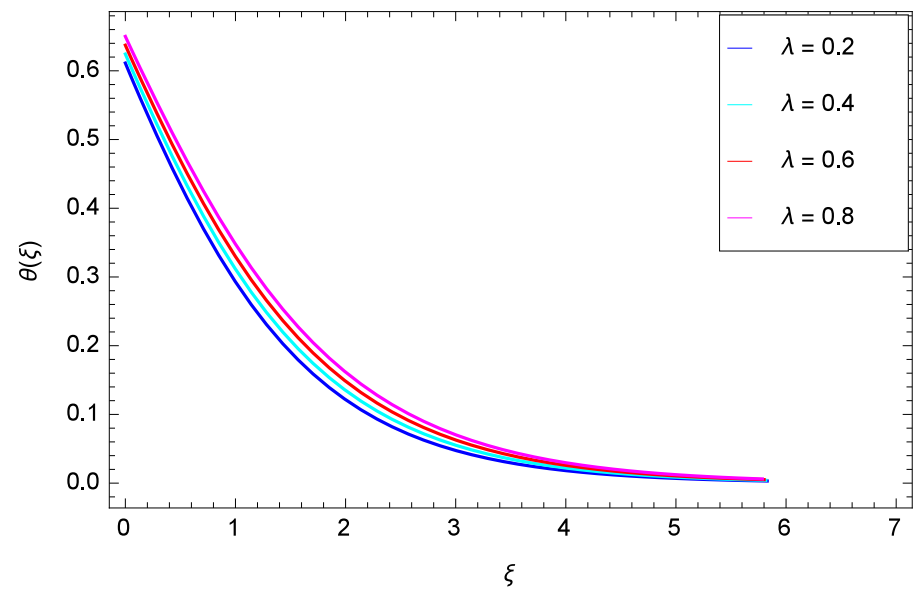

Figure 11. Impact of the heat dissipation parameter $\lambda$ on temperature $\theta(\zeta)$. 


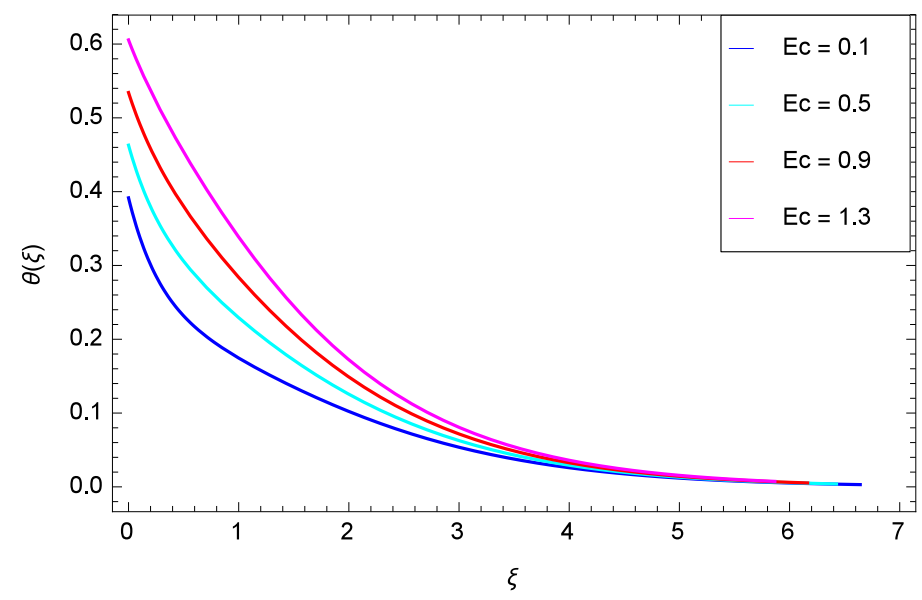

Figure 12. Impact of the Eckert number $E c$ on temperature $\theta(\zeta)$.

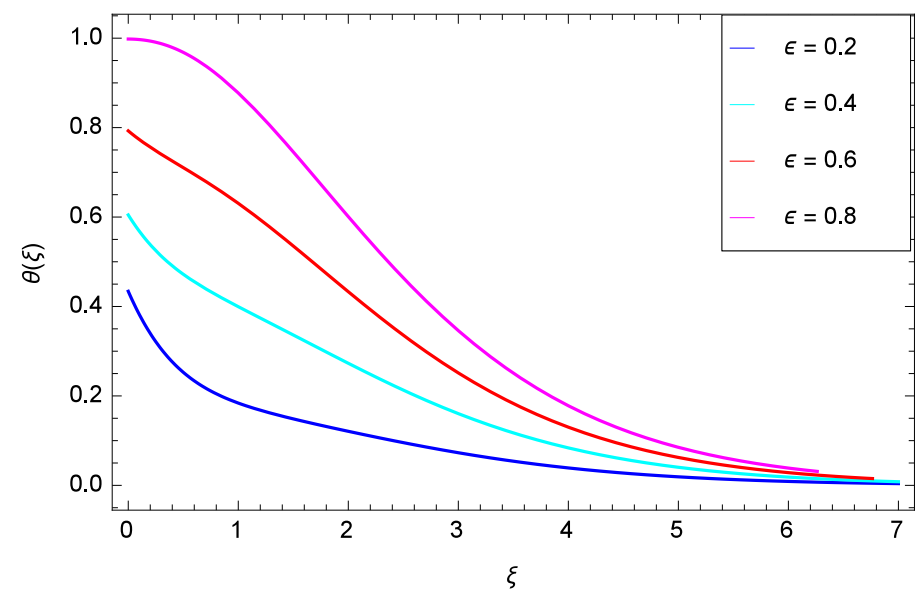

Figure 13. Impact of the curie temperature parameter $\varepsilon$ on temperature $\theta(\zeta)$.

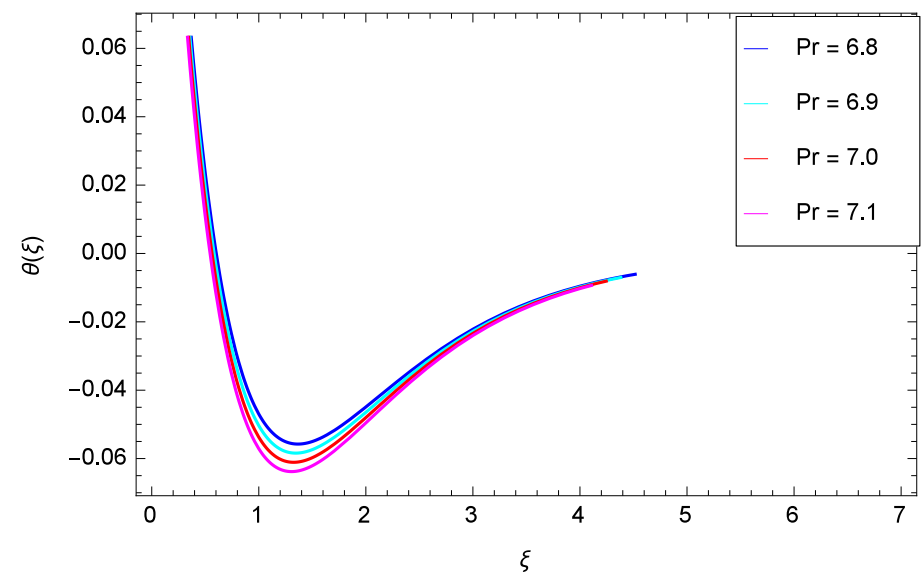

Figure 14. Impact of the Prandtl number $\operatorname{Pr}$ on temperature $\theta(\zeta)$. 


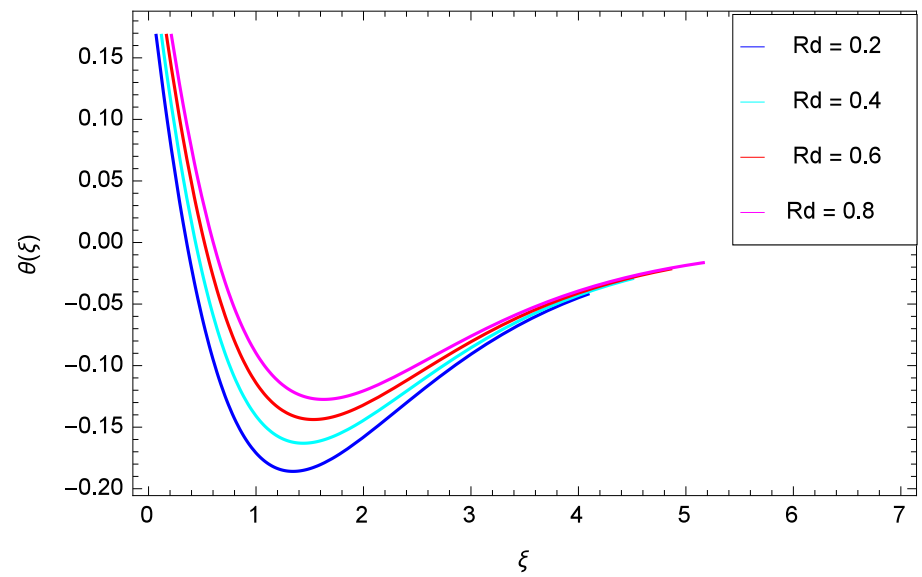

Figure 15. Impact of the thermal radiation parameter $R d$ on temperature $\theta(\zeta)$.

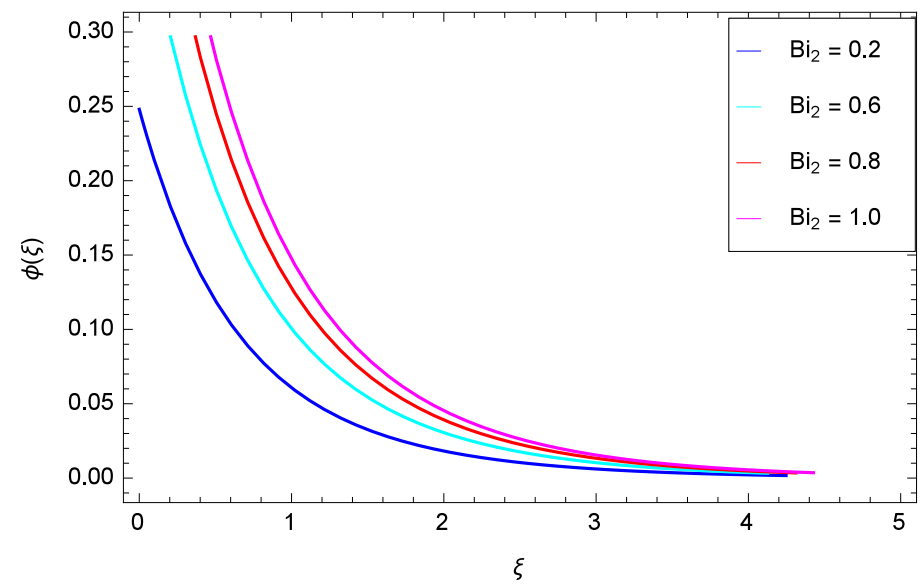

Figure 16. Impact of the solutal Biot number $B i_{2}$ on concentration $\phi(\zeta)$.

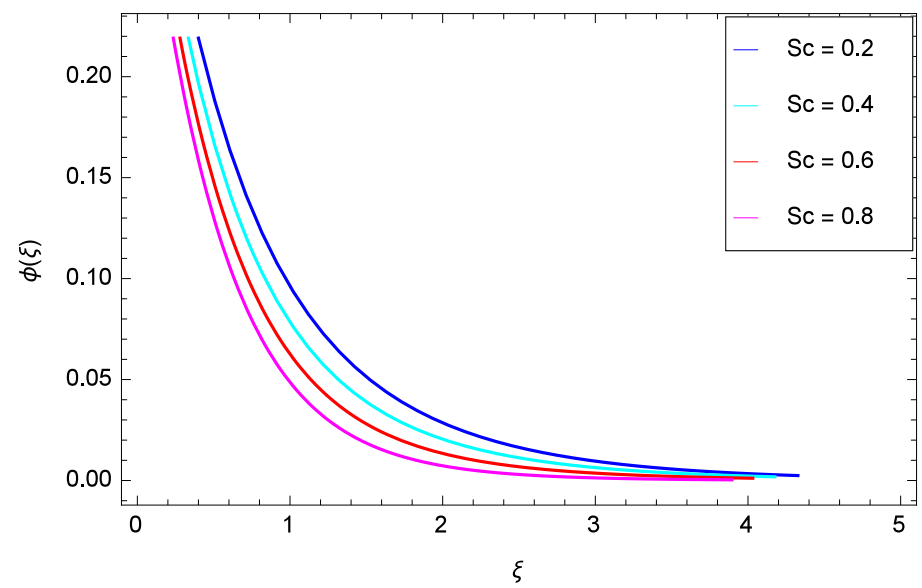

Figure 17. Impact of the Schmidt number $S c$ on concentration $\phi(\zeta)$. 


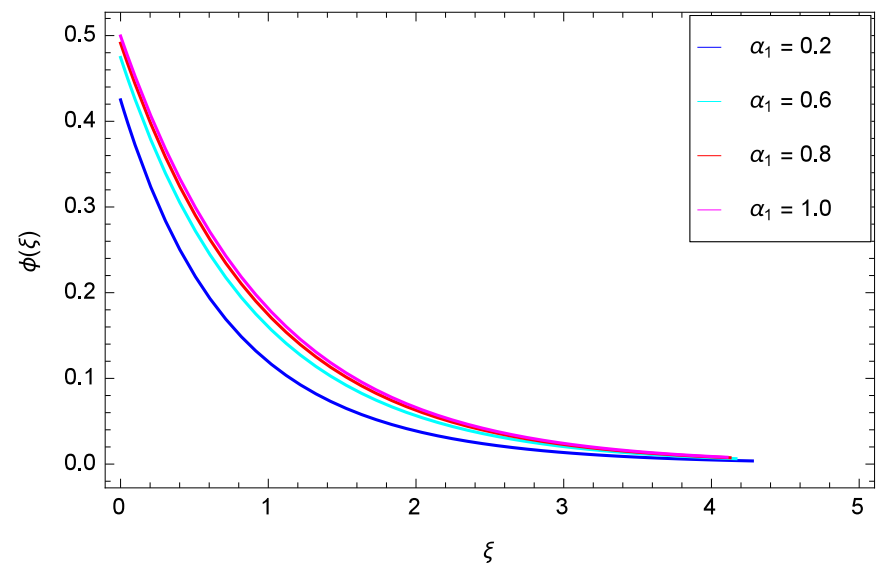

Figure 18. Impact of the curvature parameter $\alpha_{1}$ on concentration $\phi(\zeta)$.

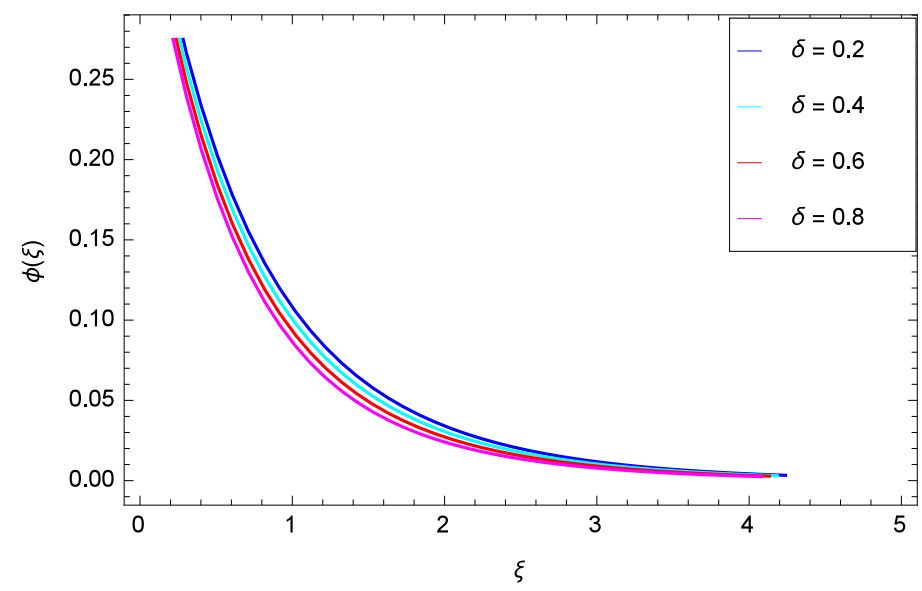

Figure 19. Impact of the chemical reaction parameter $\delta$ on concentration $\phi(\zeta)$.

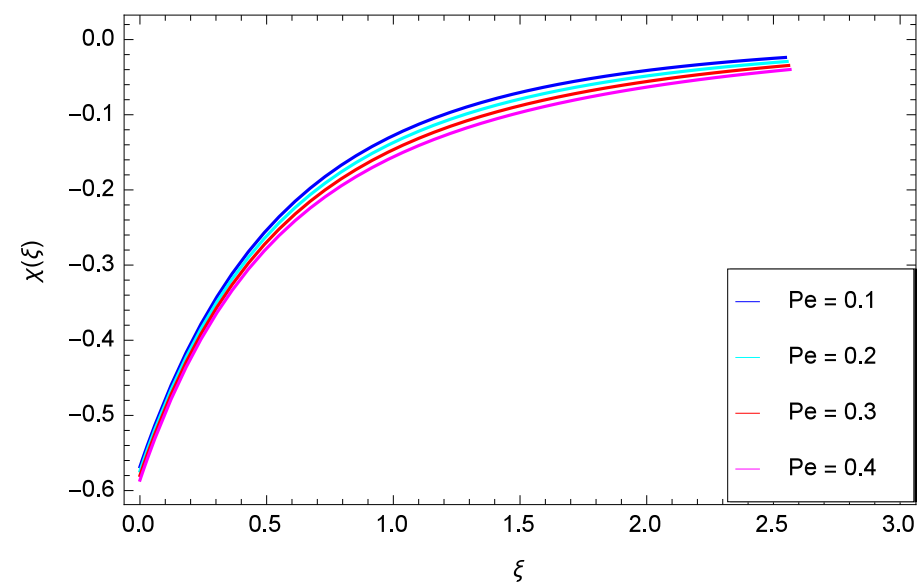

Figure 20. Impact of the Peclet number Pe on motile microorganism $\chi(\zeta)$. 


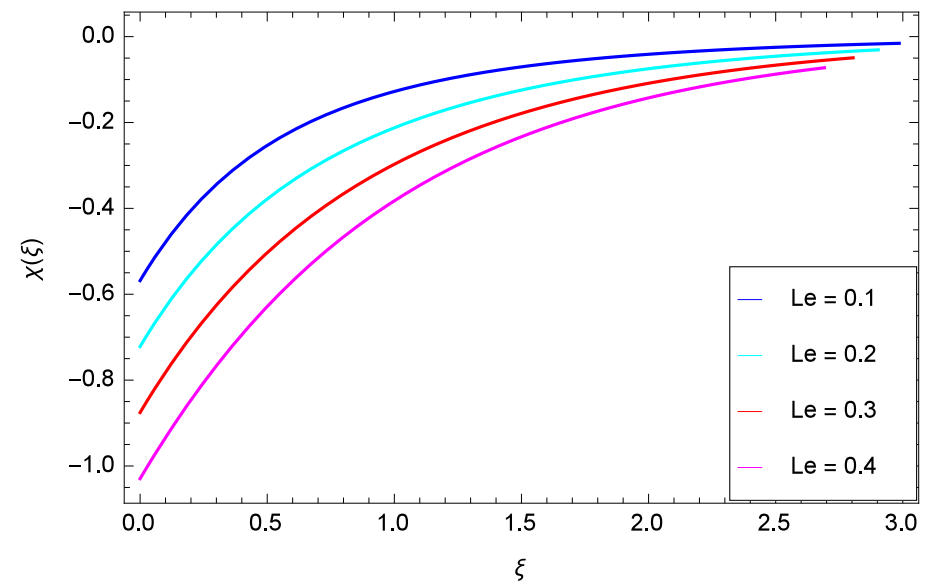

Figure 21. Impact of the Lewis number Le on motile microorganism $\chi(\zeta)$.

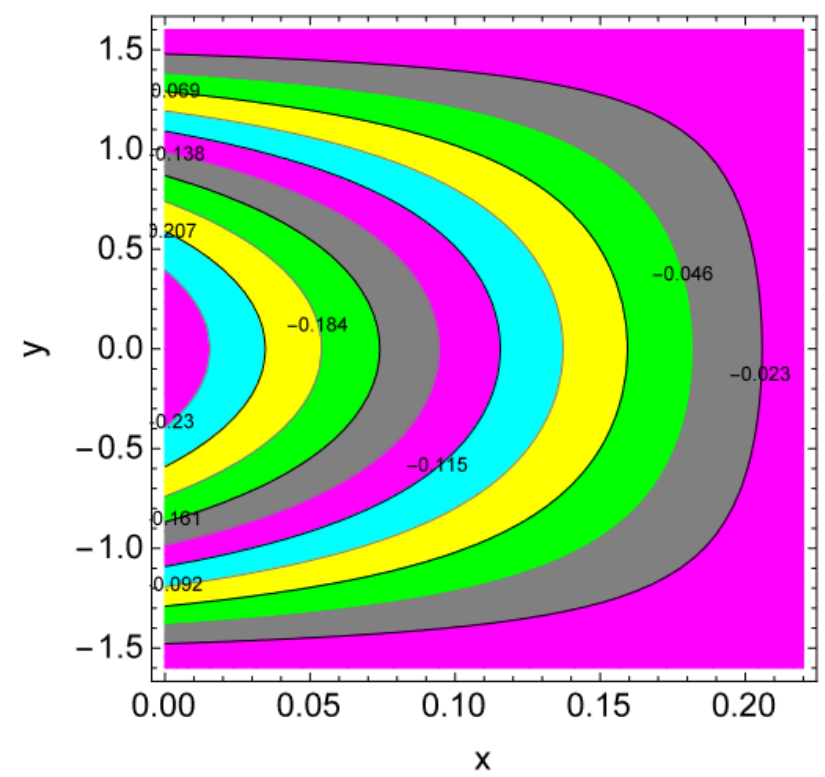

Figure 22. Behavior of streamlines for $\zeta=0.1$.

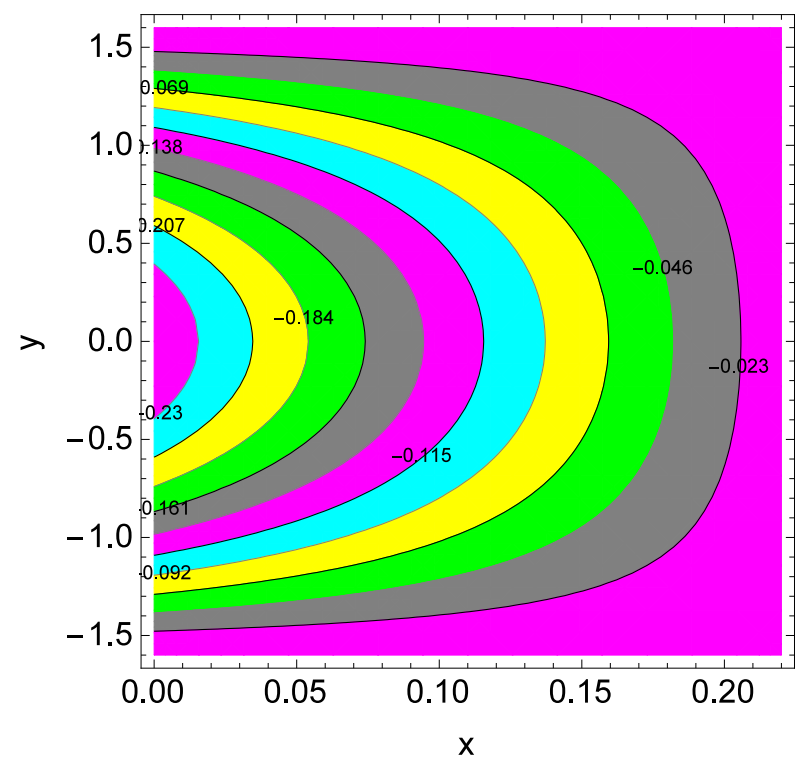

Figure 23. Behavior of streamlines for $\zeta=0.3$. 
Table 1. Numerical values of skin friction coefficient with varying values of the parameters $\beta, \alpha_{1}, P_{1}, L i, N n_{1}, N n_{2}$.

\begin{tabular}{|c|c|c|c|c|c|c|}
\hline$\beta$ & $\alpha_{1}$ & $P_{1}$ & $L i$ & $N n_{1}$ & $\mathrm{~N} n_{2}$ & $-C_{f}$ \\
\hline 0.3 & 0.4 & 0.3 & 0.3 & 0.5 & 0.5 & 1.24238 \\
\hline 0.7 & & & & & & 1.24698 \\
\hline \multirow[t]{16}{*}{1.1} & & & & & & 1.25158 \\
\hline & 0.7 & & & & & 1.24467 \\
\hline & 1.0 & & & & & 1.23511 \\
\hline & 1.3 & & & & & 1.22220 \\
\hline & & 0.6 & & & & 1.33715 \\
\hline & & 0.9 & & & & 1.43382 \\
\hline & & 1.2 & & & & 1.53241 \\
\hline & & & 0.6 & & & 1.28757 \\
\hline & & & 0.9 & & & 1.33233 \\
\hline & & & 1.2 & & & 1.37976 \\
\hline & & & & 1.0 & & 1.19970 \\
\hline & & & & 1.5 & & 1.16111 \\
\hline & & & & 2.0 & & 1.12627 \\
\hline & & & & & 1.0 & 1.20631 \\
\hline & & & & & 1.5 & 1.17235 \\
\hline & & & & & 2.0 & 1.14041 \\
\hline
\end{tabular}

Table 2. Comparative numerical values of the skin friction coefficient with published result with changing values of $\alpha_{1}$ with $P_{1}=L i=\beta=A_{1}=N n_{1}=N n_{2}=0$.

\begin{tabular}{ccc}
\hline$\alpha_{\mathbf{1}}$ & Published Work [66] & Present Work \\
\hline 5 & 0.7577 & 0.7569 \\
10 & 0.8735 & 0.8736 \\
15 & 0.9357 & 0.9357 \\
\hline
\end{tabular}

Table 3. Numerical values of Nusselt number with varying values of the parameters $\beta, \alpha_{1}, \lambda, \operatorname{Pr}, R d, \varepsilon, E c, N n_{1}, N n_{2}$.

\begin{tabular}{|c|c|c|c|c|c|c|c|c|c|}
\hline$\alpha_{1}$ & $\lambda$ & $\beta$ & $P r$ & $R d$ & $\varepsilon$ & $E c$ & $N n_{1}$ & $N n_{2}$ & $N u$ \\
\hline $\begin{array}{l}0.4 \\
0.7 \\
1.0\end{array}$ & $\begin{array}{l}0.3 \\
\\
0.8 \\
1.3 \\
1.8\end{array}$ & $\begin{array}{l}0.7 \\
1.1 \\
1.5\end{array}$ & $\begin{array}{c}6.9 \\
10.0 \\
10.11\end{array}$ & $\begin{array}{l}0.6 \\
0.8 \\
1.0\end{array}$ & $\begin{array}{l}0.4 \\
0.7 \\
1.0\end{array}$ & $\begin{array}{l}0.4 \\
0.7 \\
1.0\end{array}$ & $\begin{array}{l}1.0 \\
1.5 \\
2.0\end{array}$ & $\begin{array}{l}1.0 \\
1.5 \\
2.0\end{array}$ & $\begin{array}{l}0.331075 \\
0.330973 \\
0.330021 \\
0.332224 \\
0.332115 \\
0.332006 \\
0.332245 \\
0.332158 \\
0.332071 \\
0.333827 \\
0.335319 \\
0.336809 \\
0.379260 \\
0.426063 \\
0.472745 \\
0.332614 \\
0.332896 \\
0.333177 \\
0.334739 \\
0.337145 \\
0.339552 \\
0.332088 \\
0.331853 \\
0.331625 \\
0.333376 \\
0.334405 \\
0.335419\end{array}$ \\
\hline
\end{tabular}


Table 4. Numerical values of Sherwood number with varying values of the parameters $\alpha_{1}, S c, \delta$.

\begin{tabular}{cccc}
\hline$\alpha_{1}$ & $S c$ & $\delta$ & $S h$ \\
\hline 0.4 & 0.3 & 0.1 & 0.23643 \\
0.7 & & & 0.23658 \\
1.0 & 0.8 & & 0.23661 \\
& 1.3 & & 0.23775 \\
& 1.8 & 0.5 & 0.238104 \\
& & 0.9 & 0.238455 \\
& & 1.3 & 0.238244 \\
& & & 0.239083 \\
\hline
\end{tabular}

\section{Conclusions}

The Darcy-Forchheimer hydromagnetic flow of thixotropic nanofluid through a curved stretching sheet with thermal radiation and chemical reaction in the presence of heat and mass transfer, gyrotactic microorganisms, and magnetic dipole is explored. The present study contributes to the findings set out below.

- The velocity decreases with increasing values of ferromagnetic parameter $\beta$ and a curvature parameter $\alpha_{1}$, while it increases with increasing values of $N n_{1}, N n_{2}$ and $P_{1}$.

- The temperature increases with increasing values of $E c, \beta$, and $R d$ and decays with increasing values of $P r$.

- The nanoparticles concentration decreases with increasing values of $S c$ and $\delta$, while it increases with increasing values of $B i_{2}$ and $\alpha_{1}$.

- The distribution of the microorganism is decreased with increasing values of $P e$ and Le.

- The non-Newtonian parameters $N n_{1}$ and $N n_{2}$ have the same decreasing effects on the skin friction coefficient, while $N n_{1}$ decreases and $N n_{2}$ increases the heat transfer rate.

Author Contributions: Conceptualization, N.S.K.; methodology, A.H.; software, N.U.; validation, A.S.; formal analysis, P.K.; investigation, Q.S.; resources, P.T.; data curation, P.T.; writing-original draft preparation, A.H.U.; writing-review and editing, U.W.H.; visualization, P.K.; supervision, N.S.K.; project administration, P.K.; funding acquisition, P.K. All authors have read and agreed to the revised version of the manuscript.

Funding: This research is funded by the Center of Excellence in Theoretical and Computational Science (TaCS-CoE), KMUTT.

Institutional Review Board Statement: Not applicable.

Informed Consent Statement: Not applicable.

Acknowledgments: This work was partially supported by the International Research Partnerships: Electrical Engineering Thai-French Research Center (EE-TFRC) between King Mongkut's University of Technology North Bangkok and Universite' de Lorraine under Grant KMUTNB-BasicR-64-17. The authors are cordially thankful to the honorable reviewers for their constructive comments to improve the quality of the paper. The first author is thankful to the Higher Education Commission (HEC) Pakistan for providing the technical and financial support. This research is supported by the Postdoctoral Fellowship from King Mongkut's University of Technology Thonburi (KMUTT), Thailand. This project is supported by the Theoretical and Computational Science (TaCS) Center under Computational and Applied Science for Smart Innovation Research Cluster (CLASSIC), Faculty of Science, KMUTT.

Conflicts of Interest: The authors declare no conflict of interest.

\section{References}

1. Ershkov, S.V. Non-stationary creeping flows for incompressible 3D Navier-Stokes equations. Eur. J. Mech. B/Fluids 2017, 61, 154-159. [CrossRef]

2. Hsiao, K.L. Stagnation electrical MHD nanofluid mixed convection with slip boundary on a stretching sheet. Appl. Therm. Eng. 2016, 98, 850-861. [CrossRef] 
3. Hsiao, K.L. Micropolar nanofluid flow with MHD and viscous dissipation effects towards a stretching sheet with multimedia feature. Int. J. Heat Mass Transf. 2017, 112, 983-990. [CrossRef]

4. Hsiao, K.L. Combined electrical MHD heat transfer thermal extrusion system using Maxwell fluid with radiative and viscous dissipation effects. Appl. Therm. Eng. 2017, 112, 1281-1288. [CrossRef]

5. Hsiao, K.L. To promote radiation electrical MHD activation energy thermal extrusion manufacturing system efficiency by using Carreau-Nanofluid with parameters control method. Energy 2017, 130, 486-499. [CrossRef]

6. de Deus, H.P.A.; Dupim, G.S. On behavior of the thixotropic fluids. Phys. Lett. A 2013, 377, 478-485. [CrossRef]

7. Hayat, T.; Waqas, M.; Shehzad, S.A.; Alsaedi, A. A model of solar radiation and Joule heating in magnetohydrodynamic (MHD) convective flow of thixotropic nanofluid. J. Mol. Liq. 2016, 215, 704-710. [CrossRef]

8. Hayat, T.; Waqas, M.; Khan, M.I.; Alsaedi, A. Analysis of thixotropic nanomaterial in a doubly stratified medium considering magnetic field effects. Int. J. Heat Mass Transf. 2016, 102, 1123-1129. [CrossRef]

9. Zubair, M.; Waqas, M.; Hayat, T.; Ayub, M.; Alsaedi, A. Simulation of nonlinear convective thixotropic liquid with CattaneoChristov heat flux. Results Phys. 2018, 8, 1023-1027. [CrossRef]

10. Khan, N.S.; Shah, Z.; Islam, S.; Khan, I.; Alkanhal, T.A.; Tlili, T. Entropy generation in MHD mixed convection non-Newtonian second-grade nanoliquid thin film flow through a porous medium with chemical reaction and stratification. Entropy 2019, 21, 139. [CrossRef]

11. Khan, N.S.; Gul, T.; Islam, S.; Khan, W. Thermophoresis and thermal radiation with heat and mass transfer in a magnetohydrodynamic thin film second-grade fluid of variable properties past a stretching sheet. Eur. Phys. J. Plus 2017, $132,11$. [CrossRef]

12. Palwasha, Z.; Khan, N.S.; Shah, Z.; Islam, S.; Bonyah, E. Study of two dimensional boundary layer thin film fluid flow with variable thermo-physical properties in three dimensions space. AIP Adv. 2018, 8, 105318. [CrossRef]

13. Khan, N.S.; Gul, T.; Islam, S.; Khan, A.; Shah, Z. Brownian motion and thermophoresis effects on MHD mixed convective thin film second-grade nanofluid flow with Hall effect and heat transfer past a stretching sheet. J. Nanofluids 2017, 6, 812-829. [CrossRef]

14. Khan, N.S.; Zuhra, S.; Shah, Z.; Bonyah, E.; Khan, W.; Islam, S. Slip flow of Eyring-Powell nanoliquid film containing graphene nanoparticles. AIP Adv. 2019, 8, 115302. [CrossRef]

15. Khan, N.S.; Gul, T.; Kumam, P.; Shah, Z.; Islam, S.; Khan, W.; Zuhra, S.; Sohail, A. Influence of inclined magnetic field on Carreau nanoliquid thin film flow and heat transfer with graphene nanoparticles. Energies 2019, 12, 1459. [CrossRef]

16. Khan, N.S. Study of two dimensional boundary layer flow of a thin film second grade fluid with variable thermo-physical properties in three dimensions space. Filomat 2019, 33, 5387-5405. [CrossRef]

17. Khan, N.S.; Zuhra, S. Boundary layer unsteady flow and heat transfer in a second grade thin film nanoliquid embedded with graphene nanoparticles past a stretching sheet. Adv. Mech. Eng. 2019, 11, 1-11. [CrossRef]

18. Khan, N.S.; Gul, T.; Islam, S.; Khan, W.; Khan, I.; Ali, L. Thin film flow of a second-grade fluid in a porous medium past a stretching sheet with heat transfer. Alex. Eng. J. 2017, 57, 1019-1031. [CrossRef]

19. Zahra, A.; Mahanthesh, B.; Basir, M.F.M.; Imtiaz, M.; Mackolil, J.; Khan, N.S.; Nabwey, H.A.; Tlili, I. Mixed radiated magneto Casson fluid flow with Arrhenius activation energy and Newtonian heating effects: Flow and sensitivity analysis. Alex. Eng. J. 2020, 57, 1019-1031.

20. Liaqat, A.; Asifa, T.; Ali, R.; Islam, S.; Gul, T.; Kumam, P.; Mukhtar, S.; Khan, N.S.; Thounthong, P. A new analytical approach for the research of thin-film flow of magneto hydrodynamic fluid in the presence of thermal conductivity and variable viscosity. ZAMM J. Appl. Math. Mech. Z. Angewwandte Math. Mech. 2020, 1-13. [CrossRef]

21. Khan, N.S.; Zuhra, S.; Shah, Z.; Bonyah, E.; Khan, W.; Islam, S.; Khan, A. Hall current and thermophoresis effects on magnetohydrodynamic mixed convective heat and mass transfer thin film flow. J. Phys. Commun. 2019, 3, 035009. [CrossRef]

22. Nield, D.A.; Bejan, A. Convection in Porous Media; Springer: New York, NY, USA, 2006; p. 3.

23. Forchheimer, P. Wasserbewegung durch boden. Z. Ver. Dtsch. Ing. 1901, 45, 1782-1788.

24. Morris, M. The Flow of Homogeneous Fluids through Porous Media; J.W. Edwards Inc.: Ann Arbor, MI, USA, $1946 ;$ p. 191.

25. Kishan, N.; Maripala, S. Thermophoresis and viscous dissipation effects on Darcy-Forchheimer MHD mixed convection in a fluid saturated porous media. Adv. Appl. Sci. Res. 2012, 3, 60-74.

26. Rauf, A.; Abbas, Z.; Shehzad, S.A.; Mushtaq, T. Thermally radiative viscous fluid flow over curved moving surface in DarcyForchheimer porous space. Commun. Theor. Phys. 2019, 71, 259. [CrossRef]

27. Jagadha, S.; Amrutha, P. MHD boundary layer flow of Darcy-Forchheimer mixed convection in a nanofluid saturated porous media with viscous dissipation. Int. J. Appl. Appl. Math. 2019, 4, 117-134.

28. Andersson, H.I.; Valnes, O.A. Flow of a heated ferrofluid over a stretching sheet in the presence of a magnetic dipole. Acta Mech. 1998, 128, 39-47. [CrossRef]

29. Hayat, T.; Ahmad, S.; Khan, M.I.; Alsaedi, A. Exploring magnetic dipole contribution on radiative flow of ferromagnetic Williamson fluid. Results Phys. 2018, 8, 545-551. [CrossRef]

30. Titus, L.R.; Abraham, A. Heat transfer in ferrofluid flow over a stretching sheet with radiation. Int. J. Eng. Res. Technol. 2014, 3, 2198-2203.

31. Kefayati, G.H. Natural convection of ferrofluid in a linearly heated cavity utilizing LBM. J. Mol. Liq. 2014, 191, 1-9. [CrossRef]

32. Afkhami, S.; Renardy, Y. Ferrofluids and magnetically guided superparamagnetic particles in flows: A review of simulations and modeling. J. Eng. Math. 2017, 107, 231-251. [CrossRef] 
33. Mabood, F.; Khan, W.A.; Ismail, A.M. MHD stagnation point flow and heat transfer impinging on stretching sheet with chemical reaction and transpiration. Chem. Eng. J. 2015, 273, 430-437. [CrossRef]

34. Narayana, P.S.; Babu, D.H. Numerical study of MHD heat and mass transfer of a Jeffrey fluid over a stretching sheet with chemical reaction and thermal radiation. J. Taiwan Inst. Chem. Eng. 2016, 59, 18-25. [CrossRef]

35. Hayat, T.; Zahir, H.; Tanveer, A.; Alsaedi, A. Influences of Hall current and chemical reaction in mixed convective peristaltic flow of Prandtl fluid. J. Magn. Magn. Mater. 2016, 407, 321-327. [CrossRef]

36. Hayat, T.; Rashid, M.; Imtiaz, M.; Alsaedi, A. MHD convective flow due to a curved surface with thermal radiation and chemical reaction. J. Mol. Liq. 2017, 225, 482-489. [CrossRef]

37. Khan, N.S.; Gul, T.; Islam, S.; Khan, I.; Alqahtani, A.M.; Alshomrani, A.S. Magnetohydrodynamic nanoliquid thin film sprayed on a stretching cylinder with heat transfer. J. Appl. Sci. 2017, 7, 271. [CrossRef]

38. Khan, N.S.; Kumam, P.; Thounthong, P. Renewable energy technology for the sustainable development of thermal system with entropy measures. Int. J. Heat Mass Transf. 2019, 145, 118713. [CrossRef]

39. Khan, N.S.; Kumam, P.; Thounthong, P. Second law analysis with effects of Arrhenius activation energy and binary chemical reaction on nanofluid flow. Sci. Rep. 2020, 10, 1226. [CrossRef] [PubMed]

40. Khan, N.S.; Shah, Q.; Bhaumik, A.; Kumam, P.; Thounthong, P.; Amiri, I. Entropy generation in bioconvection nanofluid flow between two stretchable rotating disks. Sci. Rep. 2020, 10, 4448. [CrossRef] [PubMed]

41. Khan, N.S.; Shah, Q.; Sohail, A. Dynamics with Cattaneo-Christov heat and mass flux theory of bioconvection Oldroyd-B nanofluid. Adv. Mech. Eng. 2020, 12, 1-20. [CrossRef]

42. Khan, N.S.; Shah, Q.; Sohail, A.; Kumam, P.; Thounthong, P.; Bhaumik, A.; Ullah, Z. Lorentz forces effects on the interactions of nanoparticles in emerging mechanisms with innovative approach. Symmetry 2020, 5, 1700. [CrossRef]

43. Liaqat, A.; Khan, N.S.; Ali, R.; Islam, S.; Kumam, P.; Thounthong, P. Novel insights through the computational techniques in unsteady MHD second grade fluid dynamics with oscillatory boundary conditions. Heat Transf. 2020, 50, 2502-2524. [CrossRef]

44. Chamkha, A.J.; Rashad, A.M.; Kameswaran, P.K.; Abdou, M.M. Radiation effects on natural bioconvection flow of a nanofluid containing gyrotactic microorganisms past a vertical plate with streamwise temperature variation. J. Nanofluids 2017, 6, 587-595. [CrossRef]

45. Raju, C.S.; Seep, N. Dual solutions for unsteady heat and mass transfer in bio-convection flow towards a rotating cone/plate in a rotating fluid. Int. J. Eng. Res. Afr. 2016, 20, 161-176. [CrossRef]

46. Hady, F.M.; Mahdy, A.; Mohamed, R.A.; Zaid, O.A.A. Effects of viscous dissipation on unsteady MHD thermo bioconvection boundary layer flow of a nanofluid containing gyrotactic microorganisms along a stretching sheet. World J. Mech. 2016, 6, 505-526 [CrossRef]

47. Khan, N.S. Bioconvection in second grade nanofluid flow containing nanoparticles and gyrotactic microorganisms. Braz. J. Phys. 2018, 48, 227-241. [CrossRef]

48. Ferdows, M.; Zaimi, K.; Rashad, A.M.; Nabwey, H.A. MHD bioconvection flow and heat transfer of nanofluid through an exponentially stretchable sheet. Symmetry 2020, 12, 692. [CrossRef]

49. Khan, S.U.; Al-Khaled, K.; Bhatti, M.M. Bioconvection analysis for flow of Oldroyd-B nanofluid configured by a convectively heated surface with partial slip effects. Surf. Interfaces 2021, 23, 100982. [CrossRef]

50. Majeed, A.; Zeeshan, A.; Amin, N.; Ijaz, N.; Saeed, T. Thermal analysis of radiative bioconvection magnetohydrodynamic flow comprising gyrotactic microorganism with activation energy. J. Therm. Anal. Calorim. 2021, 143, 2545-2556. [CrossRef]

51. Zuhra, S.; Khan, N.S.; Alam, A.; Islam, S.; Khan, A. Buoyancy effects on nanoliquids film flow through a porous medium with gyrotactic microorganisms and cubic autocatalysis chemical reaction. Adv. Mech. Eng. 2020, 12, 1-17. [CrossRef]

52. Palwasha, Z.; Islam, S.; Khan, N.S.; Ayaz, H. Non-Newtonian nanoliquids thin film flow through a porous medium with magnetotactic microorganisms. Appl. Nanosci. 2018, 8, 1523-1544. [CrossRef]

53. Khan, N.S. Mixed convection in MHD second grade nanofluid flow through a porous medium containing nanoparticles and gyrotactic microorganisms with chemical reaction. Filomat 2019, 33, 4627-4653. [CrossRef]

54. Zuhra, S.; Khan, N.S.; Shah, Z.; Islam, Z.; Bonyah, E. Simulation of bioconvection in the suspension of second grade nanofluid containing nanoparticles and gyrotactic microorganisms. AIP Adv. 2018, 8, 105210. [CrossRef]

55. Khan, N.S.; Shah, Z.; Shutaywi, M.; Kumam, P.; Thounthong, P. A comprehensive study to the assessment of Arrhenius activation energy and binary chemical reaction in swirling flow. Sci. Rep. 2020, 10, 7868. [CrossRef] [PubMed]

56. Khan, N.S.; Gul, T.; Khan, M.A.; Bonyah, E.; Islam, S. Mixed convection in gravity-driven thin film non-Newtonian nanofluids flow with gyrotactic microorganisms. Results Phys. 2017, 7, 4033-4049. [CrossRef]

57. Liao, S.J. An explicit, totally analytic approximate solution for Blasius' viscous flow problems. Int. J. Non-Linear Mech. 1999, 34, 759-778. [CrossRef]

58. Liao, S. Beyond Perturbation: Introduction to the Homotopy Analysis Method; CRC Press: Boca Raton, FL, USA, 2003.

59. Liao, S.J. Homotopy Analysis Method in Nonlinear Differential Equations; Higher Education Press: Beijing, China; Springer: Berlin/Heidelberg, Germany, 2012.

60. Shah, Z.; Islam, S.; Gul, T.; Bonyah, E.; Khan, M.A. The electrical MHD and hall current impact on micropolar nanofluid flow between rotating parallel plates. Results Phys. 2018, 9, 1201-1214. [CrossRef]

61. Zuhra, S.; Khan, N.S.; Khan, M.A.; Islam, S.; Khan, W.; Bonyah, E. Flow and heat transfer in water based liquid film fluids dispensed with graphene nanoparticles. Result Phys. 2018, 8, 1143-1157. [CrossRef] 
62. Usman, A.H.; Khan, N.S.; Humphries, U.W.; Shah, Z.; Kumam, P.; Khan, W.; Khan, A.; Rano, S.A.; Ullah, Z. Development of dynamic model and analytical analysis for the diffusion of different species in non-Newtonian nanofluid swirling flow. Front. Phys. 2021, 8, 616790. [CrossRef]

63. Zuhra, S.; Khan, N.S.; Islam, S. Magnetohydrodynamic second-grade nanofluid flow containing nanoparticles and gyrotactic microorganisms. Comput. Appl. Math. 2018, 37, 6332-6358. [CrossRef]

64. Usman, A.H.; Humphries, U.W.; Kumam, P.; Shah, Z.; Thounthong, P. Double diffusion non-isothermal thermo-convective flow of couple stress micropolar nanofluid flow in a Hall MHD generator system. IEEE Access 2020, 8, 78821-78835. [CrossRef]

65. Ershkov, S.V.; Leshchenko, D. Dynamics of a charged particle in electromagnetic field with joule effect. Rom. Rep. Phys. 2020, $72,120$.

66. Hayat, T.; Sajjad, R.; Ellahi, R.; Alsaedi, A.; Muhammad, T. Homogeneous-heterogeneous reactions in MHD flow of micropolar fluid by a curved stretching surface. J. Mol. Liq. 2017, 240, 209-220. [CrossRef] 